\title{
Magnetoelastic Principal Parametric Resonance of a Rotating Electroconductive Circular Plate
}

\author{
Zhe Li, ${ }^{1,2}$ Yu-da Hu, ${ }^{1,2}$ and Jing $\mathrm{Li}^{2,3}$ \\ ${ }^{1}$ School of Civil Engineering and Mechanics, Yanshan University, Qinhuangdao, Hebei 066004, China \\ ${ }^{2}$ Key Laboratory of Mechanical Reliability for Heavy Equipment and Large Structures of Hebei Province, \\ Yanshan University, Qinhuangdao, Hebei 066004, China \\ ${ }^{3}$ Department of Basic Teaching, Tangshan University, Tangshan, Hebei 063000, China \\ Correspondence should be addressed to Yu-da Hu; huyuda03@163.com
}

Received 16 March 2016; Revised 6 December 2016; Accepted 11 January 2017; Published 14 February 2017

Academic Editor: Nuno M. Maia

Copyright (C) 2017 Zhe Li et al. This is an open access article distributed under the Creative Commons Attribution License, which permits unrestricted use, distribution, and reproduction in any medium, provided the original work is properly cited.

\begin{abstract}
Nonlinear principal parametric resonance and stability are investigated for rotating circular plate subjected to parametric excitation resulting from the time-varying speed in the magnetic field. According to the conductive rotating thin circular plate in magnetic field, the magnetoelastic parametric vibration equations of a conductive rotating thin circular plate are deduced by the use of Hamilton principle with the expressions of kinetic energy and strain energy. The axisymmetric parameter vibration differential equation of the variable-velocity rotating circular plate is obtained through the application of Galerkin integral method. Then, the method of multiple scales is applied to derive the nonlinear principal parametric resonance amplitude-frequency equation. The stability and the critical condition of stability of the plate are discussed. The influences of detuning parameter, rotation rate, and magnetic induction intensity are investigated on the principal parametric resonance behavior. The result shows that stable and unstable solutions exist when detuning parameter is negative, and the resonance amplitude can be weakened by changing the magnetic induction intensity.
\end{abstract}

\section{Introduction}

An annular or discoid rotating plate member application is widely used in aerospace, large generating units, and mechanical engineering. The change of the rotation speed in complicated circumstance which includes electric, magnetic, and force will cause large amplitude vibration and lead to the loss of stability, even damage the rotatable structures. Hence, it is important to analyze the dynamic response of varying parameters in rotatable system. The dynamic response of a nonlinear system is investigated in $[1,2]$. The decomposition and study of the equations of motion proposed are beneficial in the analysis of vibration. The nonlinear response of a base-excited slender beam carrying a lumped mass subjected to principal parametric resonance is investigated. The steady-state response and stability of the system by the method of multiple scales are obtained as discussed by Dwivedy and Kar [3]. EL-Bassiouny [4] investigated the principal parametric resonance of a single-degree-offreedom system with nonlinear two-frequency parametric and self-excitations and analyzed the influences of damping, nonlinear excitation, and self-excitation. Feng et al. [5] studied principal parametric resonances of a slender cantilever beam subjected to random excitation. Nonlinear vibration equation and many valuable conclusions were given. Feng and $\mathrm{Hu}[6]$ investigated principal parametric and internal resonance of flexible beams. By giving nonlinear modulation equations of combined vibration, they obtained the solutions and considered the problem of stability. Hashemi et al. [7] analyzed natural frequency and free vibration of rotating plates under different parameters. Hu et al. [8-11] investigated the problems of vibration and resonance of rectangular or circular thin plate in magnetic field. They discussed the influences of parameters on stability of a system. Özhan and Pakdemirli [12] considered the primary parametric resonance of the parametric excitation of nonlinear continuous 
system. They used the method of multiple scales to find an approximate analytical solution and discussed steady state solutions and their stability. Shahgholi and Khadem [13] studied the primary and parametric resonances of rotating asymmetrical shaft. They showed the influences of principal axes and external damping on the steady state response of the asymmetrical rotating shaft. The parametric vibrations of orthotropic plates with complex forms for different types of boundary conditions are studied with a novel approach in [14]. Awrejcewicz et al. [15] studied the chaotic vibrations of flexible plates of infinite length by the use of fast Fourier transforms and wavelets and illustrated the advantages of wavelet-oriented analysis of nonlinear vibrations of mechanical systems in [16]. Tang and Chen [17] studied the influences of variation speed of axially moving plates on stability. They calculated and analyzed natural frequency and modal under the method of multiple scales. Zhao et al. [18] investigated the stability of principal parametric resonance of stayedcable. They obtained the nonlinear equations of vibration under axial excitation with the method of multiple scales and presented numerical simulation. Zhou et al. [19] investigated the dynamic behavior of magneto-elastic-plastic interaction and coupling for beam-plates. They showed the influences of systemic parameters on plastic deformation.

In this study, the magnetoelastic axisymmetric parametric vibration equations of a conductive rotating thin circular plate are obtained. The problems of principal parametric resonance and stability of the variable-velocity rotating circular plate in transverse magnetic field are investigated, and amplitude-parameter curves are obtained for analyzing the influences of parameters on amplitude.

\section{The Magnetoelastic Vibration Equations of the Rotating Circular Plate}

The applied computational scheme is based on the Kirchhoff hypotheses and is treated as the model of first approximation. It is sufficient for engineering oriented analysis as it has been pointed out in [15]. According to the spinning circular thin plate in the magnetic field (Figure 1), the displacement vectors of each internal point of the circular plate in three directions in cylindrical coordinates are assumed as

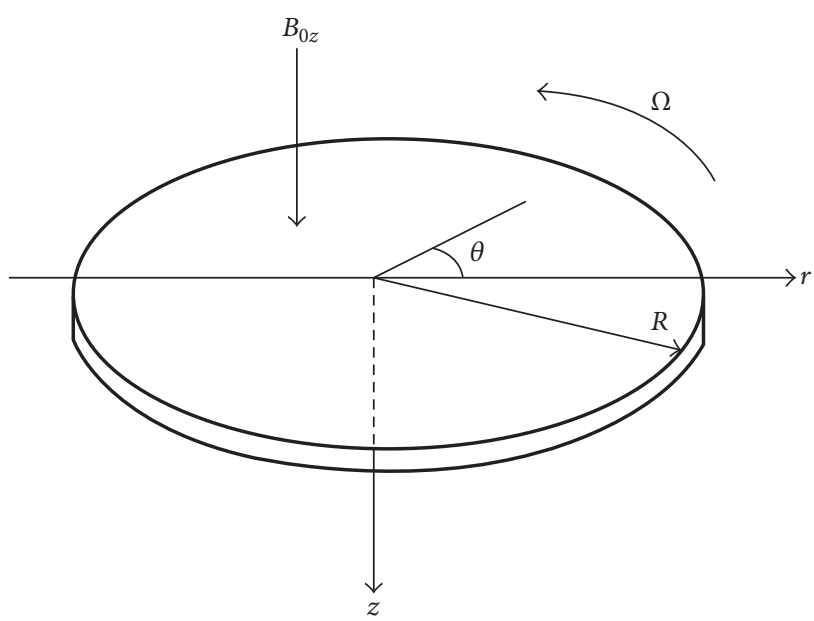

FIGURE 1: Sketch of the rotating circular plate.

$$
\begin{aligned}
& u_{r 1}(r, \theta, z, t)=u_{r}(r, \theta, t)+z u_{1}(r, \theta, t), \\
& u_{\theta 1}(r, \theta, z, t)=u_{\theta}(r, \theta, t)+z v_{1}(r, \theta, t), \\
& u_{z}(r, \theta, z, t)=w(r, \theta, t),
\end{aligned}
$$

where $u_{r}$ and $u_{\theta}$ denote the displacement in the middle plane, $w$ denotes the transverse displacement, $u_{1}=-\partial w / \partial r$ and $v_{1}=-(1 / r)(\partial w / \partial \theta)$ denote angular displacements, $r, \theta$, and $z$ denote radial, circumferential and normal coordinates, respectively, and $t$ denotes the time variable.

Velocity components of rotating circular plate, derived from (1), are as follows:

$$
\begin{aligned}
& V_{r}=\frac{d u_{r 1}}{d t}-\Omega u_{\theta 1}, \\
& V_{\theta}=\Omega\left(r+u_{r 1}\right)+\frac{d u_{\theta 1}}{d t}, \\
& V_{z}=\frac{d w}{d t}
\end{aligned}
$$

where $d / d t=\partial / \partial t+\Omega(\partial / \partial \theta)$ and $\Omega$ denotes the rotation speed. The expression of the kinetic energy can be determined as

$$
T_{k}=\frac{1}{2} \rho \int_{-h / 2}^{h / 2} \int_{0}^{R} \int_{0}^{2 \pi}\left\{\left(\frac{d u_{r 1}}{d t}-\Omega u_{\theta 1}\right)^{2}+\left[\Omega\left(r+u_{r 1}\right)+\frac{d u_{\theta 1}}{d t}\right]^{2}+\left(\frac{d w}{d t}\right)^{2}\right\} r d \theta d r d z
$$

The deformation potential caused by bending deformation can be determined as

$$
U_{\varepsilon 1}=\frac{1}{2} \int_{0}^{R} \int_{0}^{2 \pi}\left(M_{r} \kappa_{r}+M_{\theta} \kappa_{\theta}+2 M_{r \theta} \kappa_{r \theta}\right) r d r d \theta
$$

The potential energy of strain in the middle plane of the plate can be determined as

$$
U_{\varepsilon 2}=\frac{1}{2} \int_{0}^{R} \int_{0}^{2 \pi}\left(N_{r} \varepsilon_{r}+N_{\theta} \varepsilon_{\theta}+N_{r \theta} \gamma_{r \theta}\right) r d r d \theta
$$

where $M_{r}$ and $M_{\theta}$ denote bending moments, $M_{r \theta}$ denotes torque, $\kappa_{r}$ and $\kappa_{\theta}$ denote curvatures, $\kappa_{r \theta}$ denotes torsion, $N_{r}$, $N_{\theta}$, and $N_{r \theta}$ denote inertial forces in the middle plane, and $\varepsilon_{r}$, 
$\varepsilon_{\theta}$, and $\gamma_{r \theta}$ denote strains in the middle plane. It is assumed that there is transversal microdisplacement which is allowed by the displacement boundary condition, namely, virtual displacement $\delta w$. Virtual work done by the electromagnetic forces and moments $F_{r}, F_{\theta}, F_{z}, m_{r}$, and $m_{\theta}$ can be written as

$$
\begin{aligned}
\delta U_{F} & =\int_{0}^{R} \int_{0}^{2 \pi}\left[F_{r} \delta u_{r}+F_{\theta} \delta u_{\theta}+F_{z} \delta w+m_{r} \delta u_{1}\right. \\
& \left.+m_{\theta} \delta v_{1}\right] r d r d \theta .
\end{aligned}
$$

Applying the Hamilton principle, we get

$$
\int_{t_{0}}^{t_{1}}\left(\delta T_{k}-\delta U_{\varepsilon 1}-\delta U_{\varepsilon 2}+\delta U_{F}\right) d t=0
$$

Substituting (3)-(6) into (7) and considering axisymmetric vibration problem, one can compute the magnetoelastic axisymmetric vibration equation of spinning circular thin plate, which gives

$$
\begin{gathered}
\frac{\partial N_{r}}{\partial r}+\frac{N_{r}-N_{\theta}}{r}+F_{r}=\left[\frac{d^{2} u_{r}}{d t^{2}}-\Omega^{2}\left(r+u_{r}\right)\right] \rho h \\
\frac{1}{r}\left[\frac{\partial^{2}\left(r M_{r}\right)}{\partial r^{2}}-\frac{\partial M_{\theta}}{\partial r}+\frac{\partial}{\partial r}\left(r N_{r} \frac{\partial w}{\partial r}\right)\right]+\frac{1}{r} \frac{\partial\left(r m_{r}\right)}{\partial r} \\
+F_{z}=\rho h \frac{d^{2} w}{d t^{2}}-\frac{\rho h^{3}}{12}\left[\frac{1}{r} \frac{d^{2}}{d t^{2}}\left(\frac{\partial w}{\partial r}\right)\right. \\
\left.+\frac{d^{2}}{d t^{2}}\left(\frac{\partial^{2} w}{\partial r^{2}}\right)-\Omega^{2}\left(\frac{1}{r} \frac{\partial w}{\partial r}+\frac{\partial^{2} w}{\partial r^{2}}\right)\right]
\end{gathered}
$$

\section{Electromagnetic Force}

The circular plate is affected by Lorentz force in the magnetic field. The electromagnetic force per unit volume of plate is

$$
\mathbf{f}\left(f_{r}, f_{\theta}, f_{z}\right)=\mathbf{J} \times \mathbf{B}=f_{r} \mathbf{i}+f_{\theta} \mathbf{j}+f_{z} \mathbf{k},
$$

where $\mathbf{J}=\sigma_{0}(\mathbf{e}+\mathbf{V} \times \mathbf{B})$, e denotes electric field intensity, $\mathbf{V}$ denotes the velocity vector of each internal point of the thin plate, and $\sigma_{0}$ denotes the electric conductance.

Integrating (10) in the direction of the thickness, we obtain the expression of the electromagnetic forces and moments:

$$
\begin{aligned}
F_{r} & =\int_{-h / 2}^{h / 2} f_{r} d z=\sigma_{0}\left[e_{\theta}+\frac{\partial w}{\partial t} B_{0 r}-\frac{d u_{r}}{d t} B_{0 z}\right] B_{0 z} \\
& -\sigma_{0}\left[e_{z}+\frac{d u_{r}}{d t} B_{0 \theta}-\left(\Omega\left(r+u_{r}\right)+\frac{d u_{r}}{d t}\right) B_{0 r}\right] B_{0 \theta} \\
F_{z} & =\int_{-h / 2}^{h / 2} f_{z} d z=\sigma_{0}\left[\Omega\left(r+u_{r}\right) B_{0 z}-\frac{\partial w}{\partial t} B_{0 \theta}\right] B_{0 \theta} \\
& -\sigma_{0}\left[e_{\theta}+\left(\frac{\partial w}{\partial t}+\Omega \frac{\partial w}{\partial t}\right) B_{0 r}-\frac{d u_{r}}{d t} B_{0 z}\right] B_{0 r} \\
m_{r} & =\int_{-h / 2}^{h / 2} f_{r} z d z=\frac{\sigma_{0} h^{3}}{12} B_{0 z}^{2} \frac{\partial^{2} w}{\partial t \partial r} \\
& +\frac{\sigma_{0} h^{3}}{12} B_{0 \theta}^{2}\left(\frac{\partial^{2} w}{\partial t \partial r}-\frac{\Omega}{r} \frac{\partial w}{\partial r}\right)-\frac{\sigma_{0} h^{3}}{12} B_{0 r} B_{0 \theta} \Omega \frac{\partial w}{\partial r}
\end{aligned}
$$

\section{Principal Parametric Resonance of a Rotating Circular Plate in Magnetic Field}

According to the spinning circular plate in transverse magnetic field $\left(0,0, B_{0 z}\right)$ and substituting the expressions of bending moment, internal force, electromagnetic force, and electromagnetic torque into (9), the axisymmetric oscillation differential equation of the spinning circular plate in transverse magnetic field is obtained as

$$
\begin{gathered}
-D_{M}\left(\frac{\partial^{4} w}{\partial r^{4}}+\frac{2}{r} \frac{\partial^{3} w}{\partial r^{3}}-\frac{1}{r^{2}} \frac{\partial^{2} w}{\partial r^{2}}+\frac{1}{r^{3}} \frac{\partial w}{\partial r}\right)+\frac{1}{2 r} \\
\cdot D_{N}\left(\frac{\partial w}{\partial r}\right)^{3}+\frac{3}{2} D_{N}\left(\frac{\partial w}{\partial r}\right)^{2} \frac{\partial^{2} w}{\partial r^{2}}+\frac{\sigma_{0} h^{3}}{12} \\
\cdot B_{0 z}^{2}\left(\frac{1}{r} \frac{\partial^{2} w}{\partial r \partial t}+\frac{\partial^{3} w}{\partial r^{2} \partial t}\right)=\rho h \frac{\partial^{2} w}{\partial t^{2}} \\
-\frac{\rho h^{3}}{12}\left[\frac{1}{r} \frac{\partial^{3} w}{\partial r \partial t^{2}}+\frac{\partial^{4} w}{\partial r^{2} \partial t^{2}}-\Omega^{2}\left(\frac{1}{r} \frac{\partial w}{\partial r}+\frac{\partial^{2} w}{\partial r^{2}}\right)\right]
\end{gathered}
$$

where $D_{M}=E h^{3} / 12\left(1-\mu^{2}\right)$ denotes the flexural rigidity, $E$ denotes Young's modulus, $D_{N}=E h /\left(1-\mu^{2}\right)$ denotes the tensile rigidity, and $\mu$ denotes the Poisson coefficient.

We assume that an approximation to the solution of $w(r, t)$ can be expressed as

$$
\begin{aligned}
w(r, \theta, t)= & W(r) T(t) \cos (n \theta) \\
= & \sum_{n=0} r^{n} \\
& \cdot\left(\sum_{v=0,1,2, \ldots} C_{v}\left(\frac{r}{R}\right)^{2 v}\right) T_{n}(t) \cos (n \theta),
\end{aligned}
$$

where $R$ denotes the radius of the plate. The coefficients denote $C_{0}=1, C_{1}=-2, C_{2}=1$ for fixed supported conditions and $C_{0}=1, C_{1}=-(6+2 \mu) /(5+\mu), C_{2}=$ $(1+\mu) /(5+\mu)$ for simply supported conditions and we let $n=1$.

Substituting (13) into (12) and using Galerkin method, the principal parametric resonance differential equation is obtained $(n=1,2)$ :

$$
\begin{aligned}
& A_{1 n} \ddot{T}_{0}(t)+B_{1 n} \ddot{T}_{1}(t)+A_{2 n} \dot{T}_{0}(t)+B_{2 n} \dot{T}_{1}(t) \\
& +\left(A_{3 n}+A_{4 n} \Omega^{2}\right) T_{0}(t)-\left(B_{3 n}+B_{4 n} \Omega^{2}\right) T_{1}(t) \\
& \quad+A_{5 n} T_{0}^{3}(t)+B_{5 n} T_{1}^{3}(t)+A_{6 n} T_{0}^{2}(t) T_{1}(t) \\
& \quad+B_{6 n} T_{0}(t) T_{1}^{2}(t)=0,
\end{aligned}
$$

where 


$$
\begin{aligned}
& A_{1 n}=\int_{0}^{R} \int_{0}^{2 \pi}\left[\left(\rho h-\frac{\rho h^{3}}{3} \frac{C_{1}}{R^{2}}\right)+\left(\rho h \frac{C_{1}}{R^{2}}-\frac{4 \rho h^{3}}{3} \frac{C_{2}}{R^{4}}\right) r^{2}+\rho h \frac{C_{2}}{R^{4}} r^{4}\right] W \cos ^{n-1}(\theta) r^{n} d \theta d r, \\
& B_{1 n}=\int_{0}^{R} \int_{0}^{2 \pi}\left[\left(\rho h-\frac{2 \rho h^{3}}{3} \frac{C_{1}}{R^{2}}\right) r+\left(\rho h \frac{C_{1}}{R^{2}}-2 \rho h^{3} \frac{C_{2}}{R^{4}}\right) r^{3}+\rho h \frac{C_{2}}{R^{4}} r^{5}\right] W \cos ^{n}(\theta) r^{n} d \theta d r, \\
& A_{2 n}=-\int_{0}^{R} \int_{0}^{2 \pi} \frac{\sigma_{0} h^{3}}{12} B_{0 z}^{2}\left(4 \frac{C_{1}}{R^{2}}+16 \frac{C_{2}}{R^{4}} r^{2}\right) W \cos ^{n-1}(\theta) r^{n} d \theta d r \\
& B_{2 n}=-\int_{0}^{R} \int_{0}^{2 \pi} \frac{\sigma_{0} h^{3}}{12} B_{0 z}^{2}\left(8 \frac{C_{1}}{R^{2}} r+24 \frac{C_{2}}{R^{4}} r^{3}\right) W \cos ^{n}(\theta) r^{n} d \theta d r \\
& A_{3 n}=\int_{0}^{R} \int_{0}^{2 \pi} 64 D_{M} \frac{C_{2}}{R^{4}} W \cos ^{n-1}(\theta) r^{n} d \theta d r \\
& B_{3 n}=\int_{0}^{R} \int_{0}^{2 \pi} D_{M}\left(\frac{1}{r^{3}}+192 \frac{C_{2}}{R^{4}} r\right) W \cos ^{n}(\theta) r^{n} d \theta d r \\
& A_{4 n}=\int_{0}^{R} \int_{0}^{2 \pi} \frac{\rho h^{3}}{12}\left(4 \frac{C_{1}}{R^{2}}+16 \frac{C_{2}}{R^{4}}\right) W \cos ^{n-1}(\theta) r^{n} d \theta d r \\
& B_{4 n}=\int_{0}^{R} \int_{0}^{2 \pi} \frac{\rho h^{3}}{12}\left(8 \frac{C_{1}}{R^{2}} r+24 \frac{C_{2}}{R^{4}} r^{3}\right) W \cos ^{n}(\theta) r^{n} d \theta d r \\
& A_{5 n}=-\int_{0}^{R} \int_{0}^{2 \pi} D_{N}\left[\frac{1}{2 r}\left(2 \frac{C_{1}}{R^{2}} r+4 \frac{C_{2}}{R^{4}} r^{3}\right)^{3}+\frac{3}{2}\left(2 \frac{C_{1}}{R^{2}} r+4 \frac{C_{2}}{R^{4}} r^{3}\right)^{2}\left(2 \frac{C_{1}}{R^{2}}+12 \frac{C_{2}}{R^{4}}\right) r^{2}\right] W \cos ^{n-1}(\theta) r^{n} d \theta d r \\
& B_{5 n}=-\int_{0}^{R} \int_{0}^{2 \pi} D_{N}\left[\frac{1}{2 r}\left(1+3 \frac{C_{1}}{R^{2}} r^{2}+5 \frac{C_{2}}{R^{4}} r^{4}\right)^{3} \cos ^{3}(\theta)+\frac{3}{2}\left(1+3 \frac{C_{1}}{R^{2}} r^{2}+5 \frac{C_{2}}{R^{4}} r^{4}\right)\left(6 \frac{C_{1}}{R^{2}} r+20 \frac{C_{2}}{R^{4}} r^{3}\right) \cos ^{3}(\theta)\right. \\
& -\frac{1}{2 r^{2}}\left(1+3 \frac{C_{1}}{R^{2}} r^{2}+5 \frac{C_{2}}{R^{4}} r^{4}\right)^{2}\left(r+\frac{C_{1}}{R^{2}} r^{3}+\frac{C_{2}}{R^{4}} r^{5}\right) \cdot \cos ^{3}(\theta)+\frac{2}{r^{2}}\left(r+\frac{C_{1}}{R^{2}} r^{3}+\frac{C_{2}}{R^{4}} r^{5}\right)\left(1+3 \frac{C_{1}}{R^{2}} r^{2}+5 \frac{C_{2}}{R^{4}} r^{4}\right)^{2} \sin ^{2}(\theta) \\
& \cdot \cos (\theta)-\frac{1}{2 r^{3}}\left(r+\frac{C_{1}}{R^{2}} r^{3}+\frac{C_{2}}{R^{4}} r^{5}\right)^{2} \cdot\left(1+3 \frac{C_{1}}{R^{2}} r^{2}+5 \frac{C_{2}}{R^{4}} r^{4}\right) \sin ^{2}(\theta) \cos (\theta)+\frac{3}{2 r^{4}}\left(r+\frac{C_{1}}{R^{2}} r^{3}+\frac{C_{2}}{R^{4}} r^{5}\right)^{3} \sin ^{2}(\theta) \\
& \left.\cdot \cos (\theta)-\frac{1}{2 r^{2}}\left(6 \frac{C_{1}}{R^{2}} r+20 \frac{C_{2}}{R^{4}} r^{3}\right)\left(1+3 \frac{C_{1}}{R^{2}} r^{2}+5 \frac{C_{2}}{R^{4}} r^{4}\right)^{2} \cdot \sin ^{2}(\theta) \cos (\theta)\right] W \cos ^{n}(\theta) r^{n} d \theta d r \\
& A_{6 n}=-\int_{0}^{R} \int_{0}^{2 \pi} D_{N}\left\{\frac{3}{2 r}\left(2 \frac{C_{1}}{R^{2}} r+4 \frac{C_{2}}{R^{4}} r^{3}\right)^{2}\left(1+3 \frac{C_{1}}{R^{2}} r^{2}+5 \frac{C_{2}}{R^{4}} r^{4}\right) \cos (\theta)\right. \\
& +\frac{3}{2}\left[\left(2 \frac{C_{1}}{R^{2}} r+4 \frac{C_{2}}{R^{4}} r^{3}\right)^{2} \cdot\left(6 \frac{C_{1}}{R^{2}} r+20 \frac{C_{2}}{R^{4}} r^{3}\right)+2\left(2 \frac{C_{1}}{R^{2}} r+4 \frac{C_{2}}{R^{4}} r^{3}\right)\left(1+3 \frac{C_{1}}{R^{2}} r^{2}+5 \frac{C_{2}}{R^{4}} r^{4}\right)\left(2 \frac{C_{1}}{R^{2}}+12 \frac{C_{2}}{R^{4}} r^{2}\right)\right] \cos (\theta) \\
& \left.-\frac{1}{2 r^{2}}\left(r+\frac{C_{1}}{R^{2}} r^{3}+\frac{C_{2}}{R^{4}} r^{5}\right)\left(2 \frac{C_{1}}{R^{2}} r+4 \frac{C_{2}}{R^{4}} r^{3}\right)^{2} \cdot \cos (\theta)\right\} W \cos ^{n-1}(\theta) r^{n} d \theta d r \\
& B_{6 n}=-\int_{0}^{R} \int_{0}^{2 \pi} D_{N}\left\{\frac{3}{2 r}\left(2 \frac{C_{1}}{R^{2}} r+4 \frac{C_{2}}{R^{4}} r^{3}\right)^{2}\left(1+3 \frac{C_{1}}{R^{2}} r^{2}+5 \frac{C_{2}}{R^{4}} r^{4}\right)^{2} \cos ^{2}(\theta)+\frac{2}{r^{2}}\left(2 \frac{C_{1}}{R^{2}} r+4 \frac{C_{2}}{R^{4}} r^{3}\right)\right. \\
& \cdot\left(r+\frac{C_{1}}{R^{2}} r^{3}+\frac{C_{2}}{R^{4}} r^{5}\right)\left(1+3 \frac{C_{1}}{R^{2}} r^{2}+5 \frac{C_{2}}{R^{4}} r^{4}\right) \sin ^{2}(\theta) \\
& +\frac{3}{2}\left[\left(1+3 \frac{C_{1}}{R^{2}} r^{2}+5 \frac{C_{2}}{R^{4}} r^{4}\right)^{2}\left(2 \frac{C_{1}}{R^{2}}+12 \frac{C_{2}}{R^{4}} r^{2}\right)+2\left(2 \frac{C_{1}}{R^{2}} r+4 \frac{C_{2}}{R^{4}} r^{3}\right)\left(6 \frac{C_{1}}{R^{2}} r+20 \frac{C_{2}}{R^{4}} r^{3}\right) \cdot\left(1+3 \frac{C_{1}}{R^{2}} r^{2}+5 \frac{C_{2}}{R^{4}} r^{4}\right)\right] \\
& \cdot \cos ^{2}(\theta)-\frac{1}{2 r^{3}}\left(r+\frac{C_{1}}{R^{2}} r^{3}+\frac{C_{2}}{R^{4}} r^{5}\right)^{2}\left(r+\frac{C_{1}}{R^{2}} r^{3}+\frac{C_{2}}{R^{4}} r^{5}\right) \cdot \sin ^{2}(\theta)-\frac{1}{r^{2}}\left(1+3 \frac{C_{1}}{R^{2}} r^{2}+5 \frac{C_{2}}{R^{4}} r^{4}\right) \cdot\left(r+\frac{C_{1}}{R^{2}} r^{3}+\frac{C_{2}}{R^{4}} r^{5}\right) \\
& \left.\cdot\left(2 \frac{C_{1}}{R^{2}} r+4 \frac{C_{2}}{R^{4}} r^{3}\right) \cos ^{2}(\theta)+\frac{1}{2 r^{2}}\left(2 \frac{C_{1}}{R^{2}}+12 \frac{C_{2}}{R^{4}} r^{2}\right)\left(1+\frac{C_{1}}{R^{2}} r^{2}+\frac{C_{2}}{R^{4}} r^{4}\right)^{2} \cdot \sin ^{2}(\theta)\right\} W \cos ^{n}(\theta) r^{n} d \theta d r \\
& W=1+\frac{C_{1}}{R^{2}} r^{2}+\frac{C_{2}}{R^{4}} r^{4}
\end{aligned}
$$


The time history diagrams of principal parametric resonance about $T_{0}(t)$ and $T_{1}(t)$ are given in Figure $2(h=8 \mathrm{~mm}$, $\left.R=0.8 \mathrm{~m}, \Omega_{1}=400 \mathrm{r} / \mathrm{min}, B_{0 z}=0.1 \mathrm{~T}, \Omega_{0}=7500 \mathrm{r} / \mathrm{min}\right)$. This figure shows that under the same conditions the firstorder amplitude $T_{0}(t)$ is greater than the second-order amplitude $T_{1}(t)$. In order to simplify the calculation, we only analyze first-order amplitude:

$$
\begin{aligned}
& A_{1} \ddot{T}_{0}(t)+A_{2} \dot{T}_{0}(t)+\left(A_{3}+A_{4} \Omega^{2}\right) T_{0}(t)-A_{5} T_{0}^{3}(t) \\
& \quad=0
\end{aligned}
$$

where

$$
\begin{aligned}
A_{1} & =\frac{\rho h}{2} R^{2}+\left(\frac{\rho h}{2} R^{2}-\frac{\rho h^{3}}{6}\right) C_{1}+\left(\frac{\rho h}{3} R^{2}-\frac{\rho h^{3}}{3}\right) \\
& \cdot C_{2}+\left(\frac{\rho h}{6} R^{2}-\frac{\rho h^{3}}{12}\right) C_{1}^{2}+\left(\frac{\rho h}{10} R^{2}-\frac{\rho h^{3}}{6}\right) C_{2}^{2} \\
& +\left(\frac{\rho h}{4} R^{2}-\frac{5 \rho h^{3}}{18}\right) C_{1} C_{2}, \\
A_{2} & =-\frac{\sigma_{0} h^{3}}{12} B_{0 z}^{2}\left(2 C_{1}+4 C_{2}+C_{1}^{2}+2 C_{2}^{2}+\frac{10}{3} C_{1} C_{2}\right), \\
A_{3} & =\frac{D_{M}}{R^{2}}\left(\frac{32}{3} C_{2}^{2}+16 C_{1} C_{2}+32 C_{2}\right), \\
A_{4} & =\frac{\rho h^{3}}{12}\left(2 C_{1}+4 C_{2}+C_{1}^{2}+2 C_{2}^{2}+\frac{10}{3} C_{1} C_{2}\right), \\
A_{5} & =\frac{D_{N}}{R^{2}}\left(\frac{8}{3} C_{1}^{4}+4 C_{1}^{3}+20 C_{1}^{3} C_{2}+\frac{264}{5} C_{1}^{2} C_{2}^{2}\right. \\
& +24 C_{1}^{2} C_{2}+48 C_{1} C_{2}^{2}+\frac{176}{3} C_{1} C_{2}^{3}+32 C_{2}^{3} \\
& \left.+\frac{160}{7} C_{2}^{4}\right) .
\end{aligned}
$$

We assume

$$
\Omega=\Omega_{0}+\Omega_{1} \cos \omega_{1} t
$$

where $\Omega_{0}$ denotes a constant term, $\Omega_{1}$ denotes the constant amplitude of disturbance term (the cosinusoid variation of the angular velocity), and $\omega_{1}$ denotes the frequency of disturbance term. The order of $\Omega_{1}$ is less than the order of $\Omega_{0}$.

Substituting (18) into (16), one can obtain

$$
\begin{aligned}
\ddot{T}_{0}(t) & +\eta_{1} \dot{T}_{0}(t)+\left[\eta_{2}+\eta_{3}\left(\Omega_{0}^{2}+\frac{1}{2} \Omega_{1}^{2}\right)\right] T_{0}(t) \\
& +\left(2 \Omega_{0} \Omega_{1} \eta_{3} \cos \omega_{1} t+\frac{\Omega_{1}^{2}}{2} \eta_{3} \cos 2 \omega_{1} t\right) T_{0}(t) \\
& -\eta_{4} T_{0}^{3}(t)=0,
\end{aligned}
$$

where $\eta_{1}=A_{2} / A_{1}, \eta_{2}=A_{3} / A_{1}, \eta_{3}=A_{4} / A_{1}$, and $\eta_{4}=$ $A_{5} / A_{1}$.
For simplification, we let $\omega_{1} t=2 \tau, q(\tau)=T_{0}(\tau) / h$ and obtain

$$
\ddot{q}+2 \widetilde{\xi} \dot{q}+\omega_{n}^{2} q+\left(\widetilde{h}_{1} \cos 2 \tau+\widetilde{h}_{2} \cos 4 \tau\right) q-\widetilde{h}_{3} q^{3}=0,
$$

where $\widetilde{\xi}=\eta_{1} / \omega_{1}, \widetilde{h}_{1}=8 \Omega_{0} \Omega_{1} \eta_{3} / \omega_{1}^{2}, \widetilde{h}_{2}=2 \Omega_{1}^{2} \eta_{3} / \omega_{1}^{2}, \widetilde{h}_{3}=$ $4 h^{2} \eta_{4} / \omega_{1}^{2}$, and $\omega_{n}^{2}=\left(4 \eta_{2}+4 \eta_{3}\left(\Omega_{0}^{2}+(1 / 2) \Omega_{1}^{2}\right)\right) / \omega_{1}^{2}$.

We introduce a small parameter $\varepsilon$. Equation (20) can be expressed as

$$
\begin{aligned}
\ddot{q}+ & 2 \varepsilon \dot{q} \dot{q}+\omega_{n}^{2} q+\left(\varepsilon h_{1} \cos 2 \tau+\varepsilon h_{2} \cos 4 \tau\right) q-\varepsilon h_{3} q^{3} \\
& =0,
\end{aligned}
$$

where $\xi=\tilde{\xi} / \varepsilon, h_{1}=\widetilde{h}_{1} / \varepsilon, h_{2}=\widetilde{h}_{2} / \varepsilon$, and $h_{3}=\widetilde{h}_{3} / \varepsilon$.

We assume

$$
\omega_{n}=1+\varepsilon \sigma,
$$

where $\sigma$ denotes the detuning parameter. By the use of the method of multiple scales [20], the solution of (21) in terms of different time scales can be expressed as

$$
q(\tau, \varepsilon)=q_{0}\left(T_{0}, T_{1}\right)+\varepsilon q_{1}\left(T_{0}, T_{1}\right) .
$$

Substituting (22)-(23) into (21) and equating the coefficients of the same order of $\varepsilon$ in both sides, the following equations are obtained:

$$
\begin{aligned}
D_{0}^{2} q_{0}+q_{0}= & 0 \\
D_{0}^{2} q_{1}+q_{1}= & -2 D_{0} D_{1} q_{0}-2 \xi D_{0} q_{0}-2 \sigma q_{0} \\
& -\left(h_{1} \cos 2 \tau+h_{2} \cos 4 \tau\right) q_{0}+h_{3} q_{0}^{3},
\end{aligned}
$$

where $D_{0}=\partial / \partial T_{0}, D_{1}=\partial / \partial T_{1}$. The solution of (24) is

$$
q_{0}=A\left(T_{1}\right) e^{\mathrm{i} T_{0}}+\bar{A}\left(T_{1}\right) e^{-\mathrm{i} T_{0}} .
$$

Substituting (26) into (25), one may obtain

$$
\begin{aligned}
D_{0}^{2} q_{1}+q_{1}= & \left(3 h_{3} A^{2} \bar{A}-2 \mathrm{i} A^{\prime}-2 \mathrm{i} \xi A-2 \sigma A\right) e^{\mathrm{i} T_{0}} \\
& +h_{3} A^{3} e^{3 \mathrm{i} T_{0}}-\frac{h_{1}}{2}\left(A e^{3 \mathrm{i} T_{0}}+\bar{A} e^{\mathrm{i} T_{0}}\right) \\
& -\frac{h_{2}}{2}\left(A e^{5 \mathrm{i} T_{0}}+\bar{A} e^{3 \mathrm{i} T_{0}}\right)+\mathrm{cc},
\end{aligned}
$$

where $A^{\prime}=\partial A / \partial T_{1}$ and $c c$ is conjugate complex number in right side.

The solvability conditions of (27) express that the coefficients of $e^{\mathrm{i} T_{0}}$ must be vanished. Then (27) has nontrivial solution which gives

$$
3 h_{3} A^{2} \bar{A}-2 \mathrm{i} A^{\prime}-2 \mathrm{i} \xi A-2 \sigma A-\frac{h_{1}}{2} \bar{A}=0 .
$$

To solve (28), $A\left(T_{1}\right)$ is written in the polar form:

$$
A=\frac{1}{2} a\left(T_{1}\right) e^{\mathrm{i} \varphi\left(T_{1}\right)},
$$



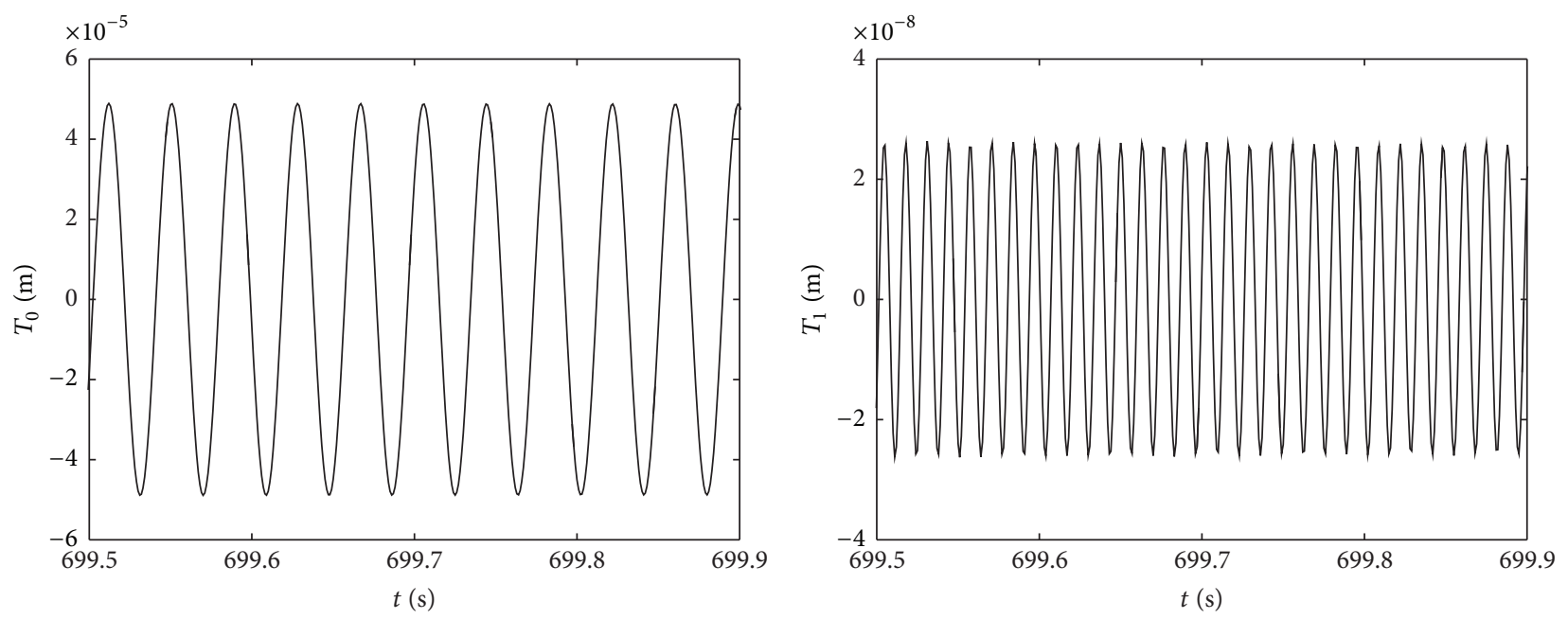

FIgURE 2: Time history diagram.

where $a$ and $\varphi$ are real numbers. Substituting (29) into (28) and separating the result into its real and imaginary parts, we obtain

$$
\begin{aligned}
& a^{\prime}=-\xi a+\frac{a h_{1}}{4} \sin 2 \varphi, \\
& \varphi^{\prime}=\sigma-\frac{3 a^{2} h_{3}}{8}+\frac{h_{1}}{4} \cos 2 \varphi .
\end{aligned}
$$

The frequency-response equation of the principal parametric resonance can be obtained by combining the equations of (30) when $a^{\prime}=0, \varphi^{\prime}=0$ :

$$
\xi^{2}+\left(\sigma-\frac{3 a^{2} h_{3}}{8}\right)^{2}=\left(\frac{h_{1}}{4}\right)^{2} .
$$

The stabilization of the steady-state solutions correspond to the stabilization of the singular points. As in (30), we let [20]

$$
\begin{aligned}
& a=a_{0}+a_{1}, \\
& \varphi=\varphi_{0}+\varphi_{1},
\end{aligned}
$$

where $a_{1}$ and $\varphi_{1}$ are small compared with $a_{0}$ and $\varphi_{0}$. Substituting (32) into (30) and linearizing the resulting equations in $a_{1}$ and $\varphi_{1}$, we obtain

$$
\begin{aligned}
& a_{1}^{\prime}=-\xi a_{1}+2\left(\frac{3 h_{3}}{8} a_{0}^{3}-\sigma a_{0}\right) \varphi_{1}, \\
& \varphi_{1}^{\prime}=-\frac{3 h_{3} a_{0}}{4} a_{1}-2 \xi \varphi_{1} .
\end{aligned}
$$

According to the stability theory, the solution is stable if the real parts of characteristic values are negative in (33). Due to $\xi>0$, the real parts of characteristic values are negative if and only if

$$
\frac{3 h_{3}}{2} a_{0}^{2}\left(\frac{3 h_{3}}{8} a_{0}^{2}-\sigma\right)>0 .
$$

As this time, the steady-state motion of rotating system is stable.

\section{Numerical Study}

For numerical study of nonlinear primary parametric resonances of rotating circular plate with simply supported conditions, the circular plate with the following parameters is used: the mass density $\rho=2670 \mathrm{~kg} / \mathrm{m}^{3}$, Young's modulus $E=71 \mathrm{GPa}$, the electric conductance $\sigma_{0}=3.63 \times 10^{7}(\Omega \cdot \mathrm{m})^{-1}$, and Poisson's ratio $\mu=0.34$. In the figures, the solid curves denote stable solution and the dotted line denotes unstable solution.

Figure 3 shows the amplitude-frequency response curves $\left(B_{0 z}=0.1 \mathrm{~T}\right)$. In order to make curves clear, the detuning parameters and amplitude values are small. It is seen that the value of amplitude $a$ decreases with the sign of the detuning parameter $\varepsilon \sigma$ changing from negative to positive. Resonance phenomenon vanishes when the amplitude $a$ changes to zero. We also notice the resonance region between the stable and unstable solutions increases with the increase of $\Omega_{1}$, $\Omega_{0}$, and radius $R$ and the decrease of thickness $h$. There is an intersection in Figure 3(a), and different regularities exist in both sides of this intersection. The stable amplitude increases with the parametric $\Omega_{0}$ decreasing at the left of intersection and decreases with the parametric $\Omega_{0}$ decreasing at the right of intersection. According to numerical conversion, we compare the first-order displacement amplitude (Figure 2) which is obtained by numerical calculation with amplitude frequency diagram (Figure 3(b)) which is obtained by amplitude frequency response equation of steady motion with given parameters and find that they are identical. It shows that the rationality of solving the resonance solution with the first order approximate expression of the multiscale method is confirmed.

Figure 4 shows the characteristic curves of relationship between the amplitude and the magnetic induction intensity $B_{0 z}\left(h=8 \mathrm{~mm}, R=1 \mathrm{~m}, \Omega_{1}=200 \mathrm{r} / \mathrm{min}\right)$. This figure shows that system has stable and unstable solutions. When the magnetic induction intensity $B_{0 z}$ is small, the change of amplitude is insensitive. From Figure 3(b), we can see 


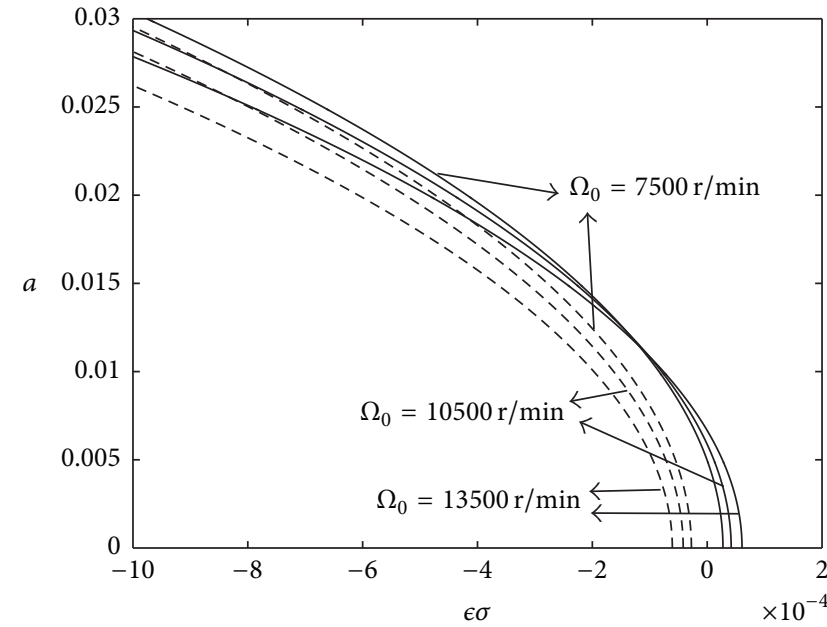

(a) $h=8 \mathrm{~mm}, R=0.8 \mathrm{~m}, \Omega_{1}=400 \mathrm{r} / \mathrm{min}$

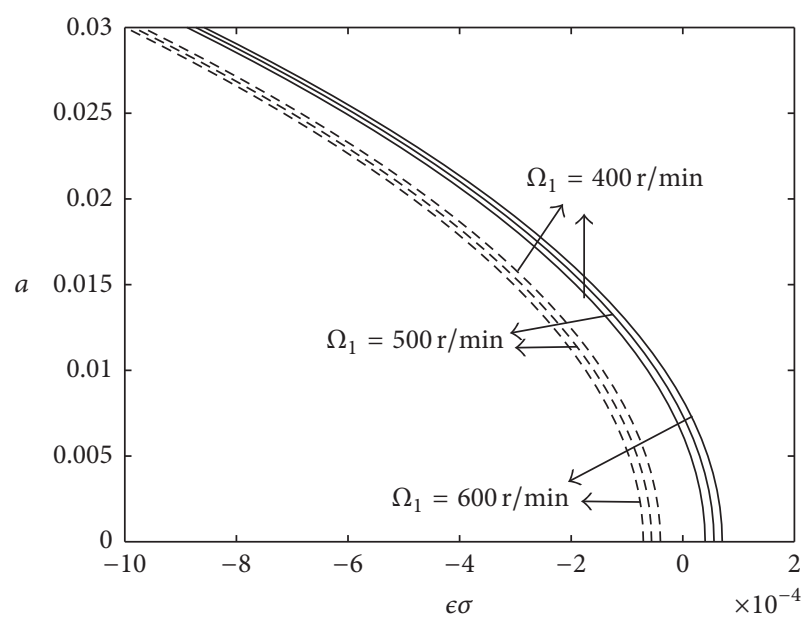

(c) $R=0.8 \mathrm{~m}, \Omega_{0}=7500 \mathrm{r} / \mathrm{min}, h=8 \mathrm{~mm}$

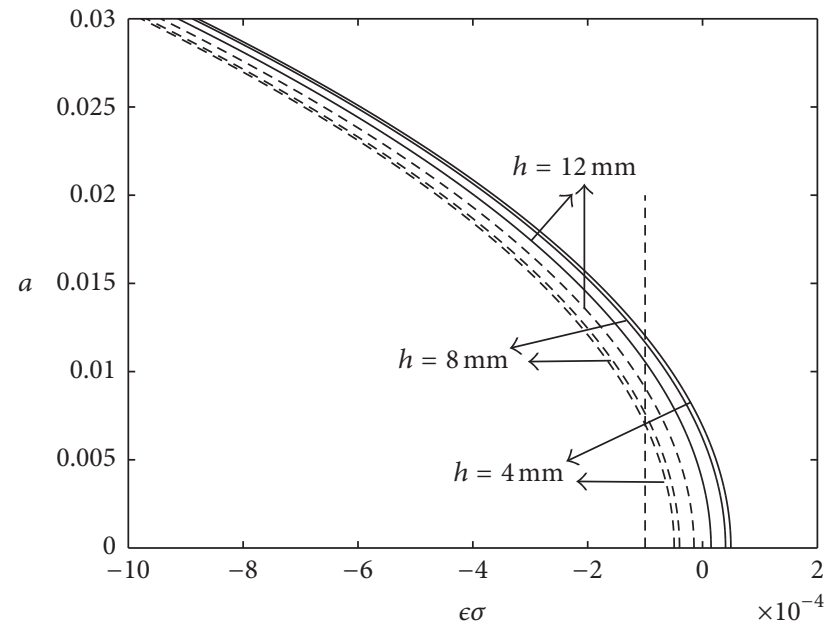

(b) $R=0.8 \mathrm{~m}, \Omega_{0}=7500 \mathrm{r} / \mathrm{min}, \Omega_{1}=400 \mathrm{r} / \mathrm{min}$

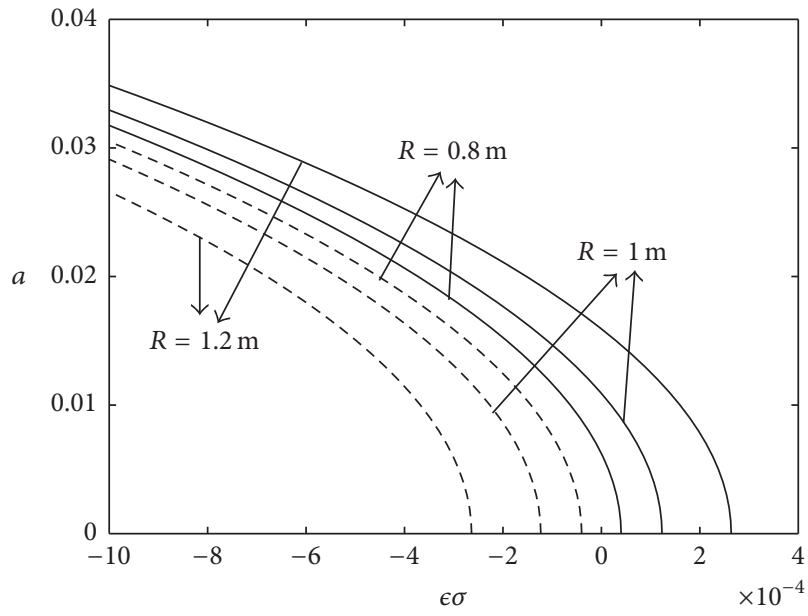

(d) $\Omega_{1}=400 \mathrm{r} / \mathrm{min}, \Omega_{0}=7500 \mathrm{r} / \mathrm{min}, h=8 \mathrm{~mm}$

FIGURE 3: Amplitude-frequency response curves.

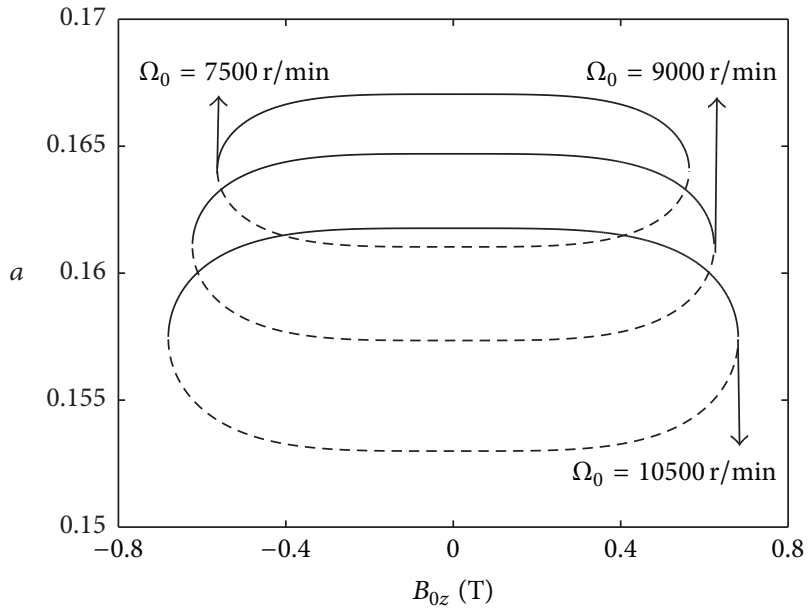

(a) $\varepsilon \sigma=-0.03$

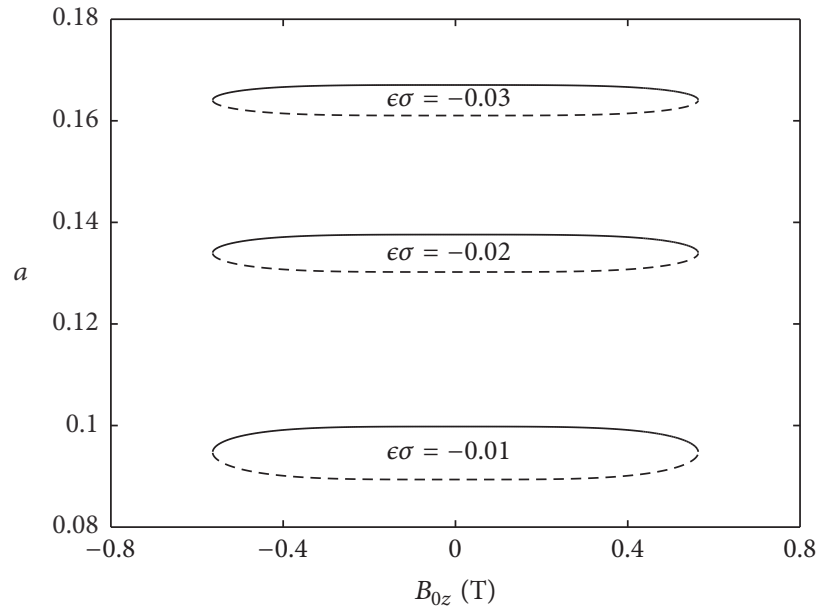

(b) $\Omega_{0}=7500 \mathrm{r} / \mathrm{min}$

FIGURE 4: The characteristic curves of amplitude-magnetic induction intensity. 


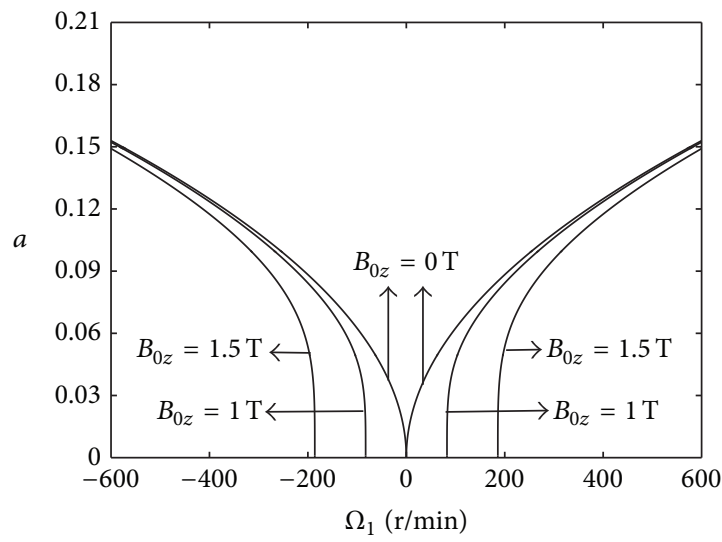

(a) $\varepsilon \sigma=0, \Omega_{0}=7500 \mathrm{r} / \mathrm{min}$

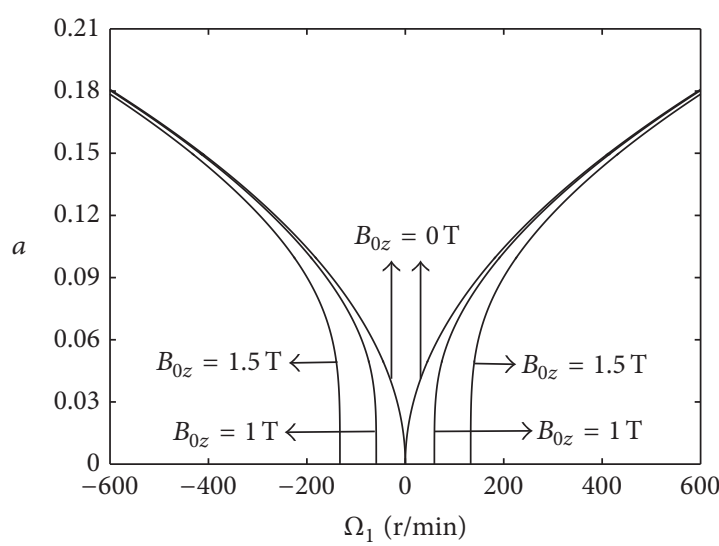

(c) $\varepsilon \sigma=0, \Omega_{0}=10500 \mathrm{r} / \mathrm{min}$

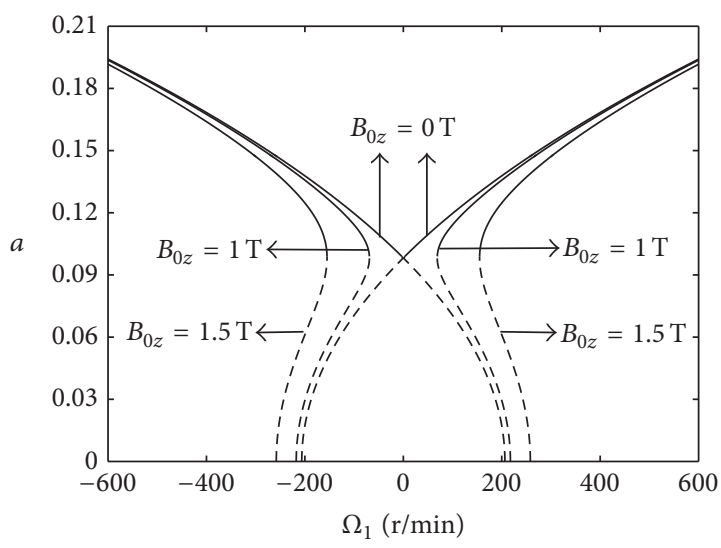

(e) $\varepsilon \sigma=-0.01, \Omega_{0}=9000 \mathrm{r} / \mathrm{min}$

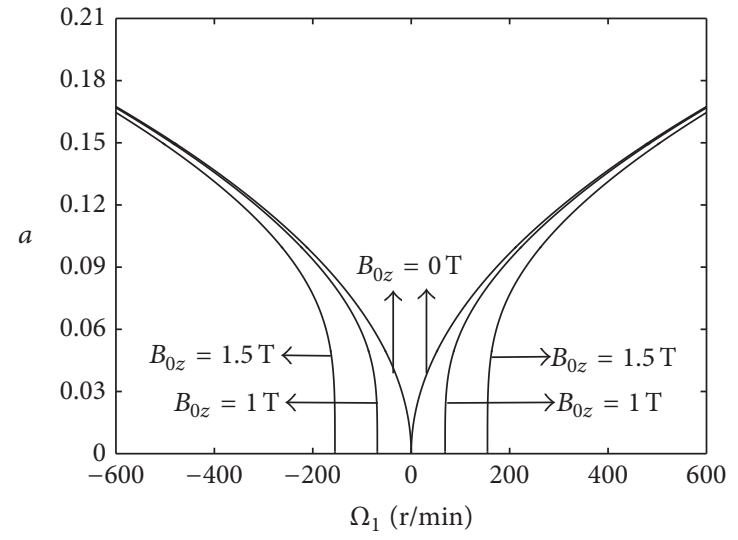

(b) $\varepsilon \sigma=0, \Omega_{0}=9000 \mathrm{r} / \mathrm{min}$

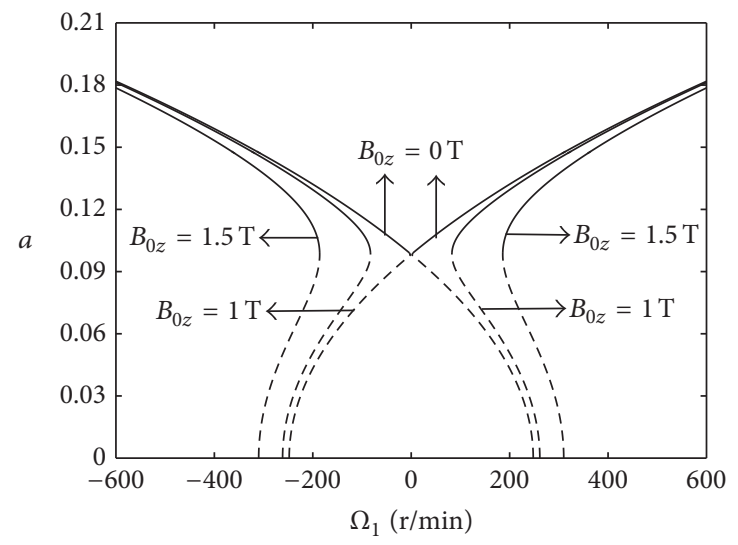

(d) $\varepsilon \sigma=-0.01, \Omega_{0}=7500 \mathrm{r} / \mathrm{min}$

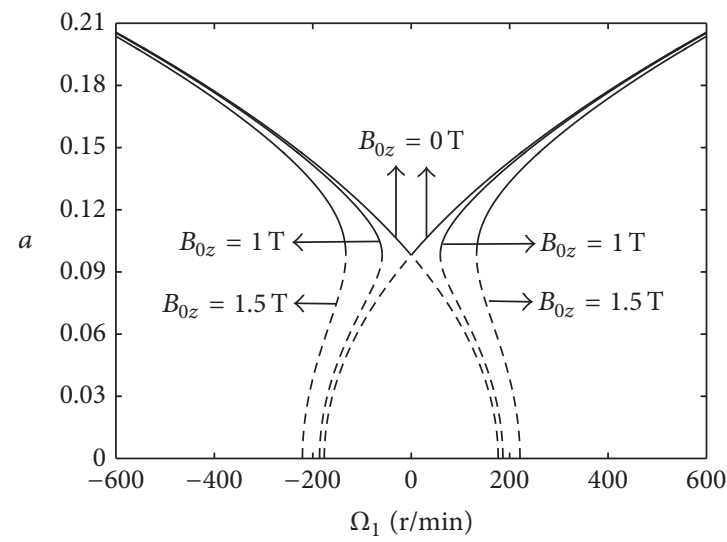

(f) $\varepsilon \sigma=-0.01, \Omega_{0}=10500 \mathrm{r} / \mathrm{min}$

FIGURE 5: The characteristic curves of resonance amplitude-amplitude of velocity disturbance term.

that the magnetic induction intensity remains constant or substantially constant with the variation of the detuning parameter when bifurcation emerges. However, saddle-node bifurcation exists and resonance vanishes when the magnetic induction intensity increases to a critical value. It means that we can attenuate the resonance phenomenon of rotating plate by changing the magnetic field intensity while other parameters are constant.
Figure 5 shows the characteristic curves of relationship between the amplitude $a$ and the amplitude of velocity disturbance term $\Omega_{1}$ with different magnetic induction intensity $B_{0 z}(h=8 \mathrm{~mm}, R=1 \mathrm{~m})$. The figure shows that unstable nontrivial solutions exist when the detuning parameter $\varepsilon \sigma=$ -0.01 , while stable nontrivial solutions exist when $\varepsilon \sigma=0$. The nonresonance region, called zero solution region, expands with the increase of $B_{0 z}$. Meanwhile, we can see that the 


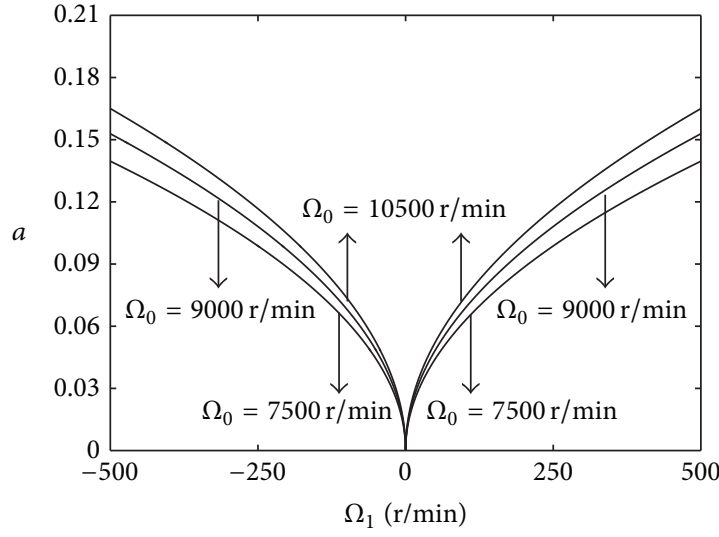

(a) $\varepsilon \sigma=0, B_{0 z}=0 \mathrm{~T}$

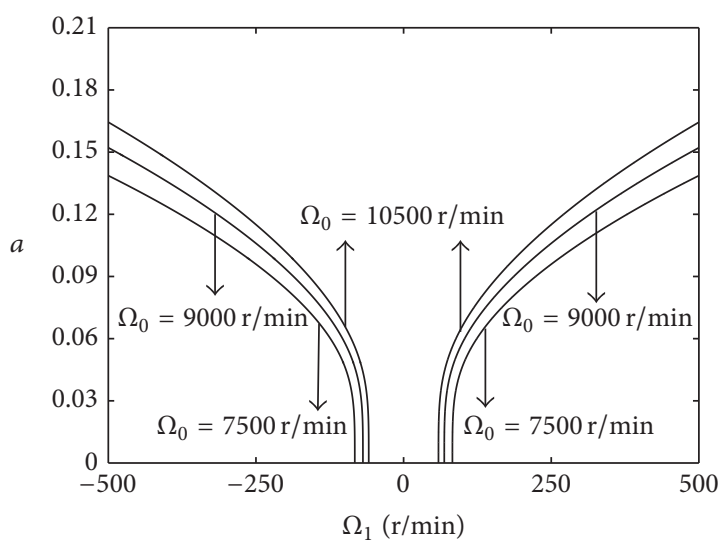

(c) $\varepsilon \sigma=0, B_{0 z}=1 \mathrm{~T}$

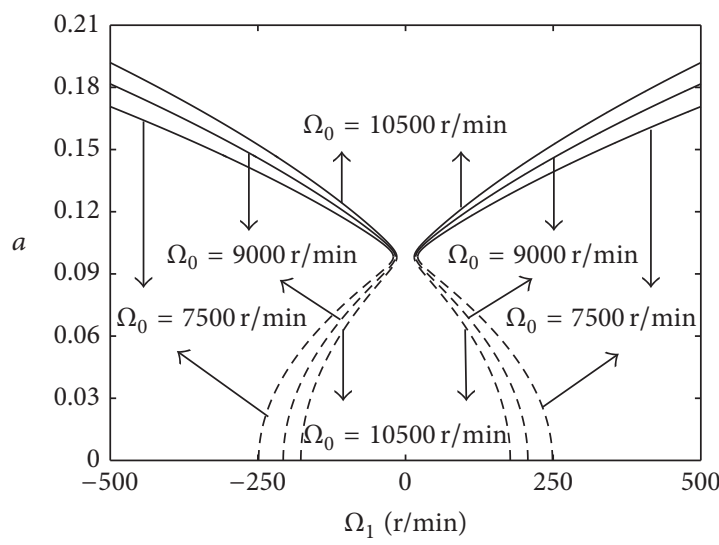

(e) $\varepsilon \sigma=-0.01, B_{0 z}=0.5 \mathrm{~T}$

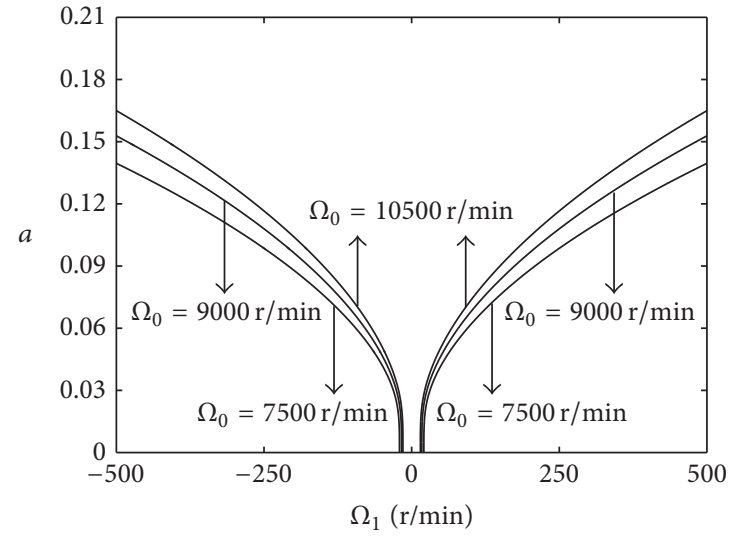

(b) $\varepsilon \sigma=0, B_{0 z}=0.5 \mathrm{~T}$

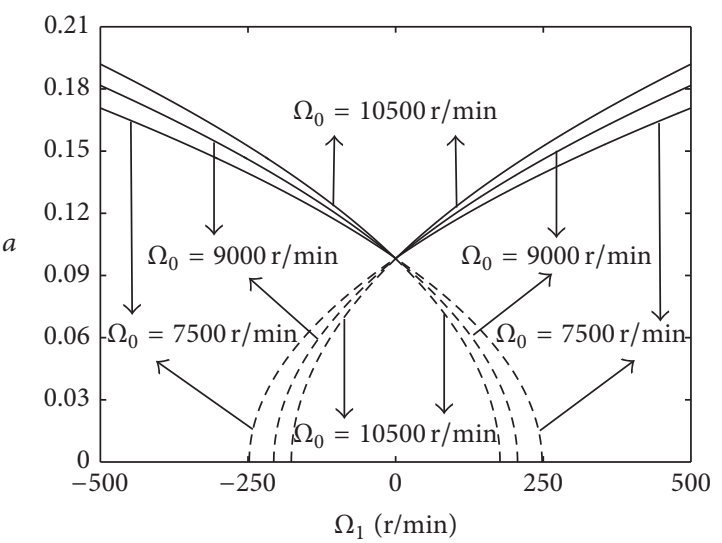

(d) $\varepsilon \sigma=-0.01, B_{0 z}=0 \mathrm{~T}$

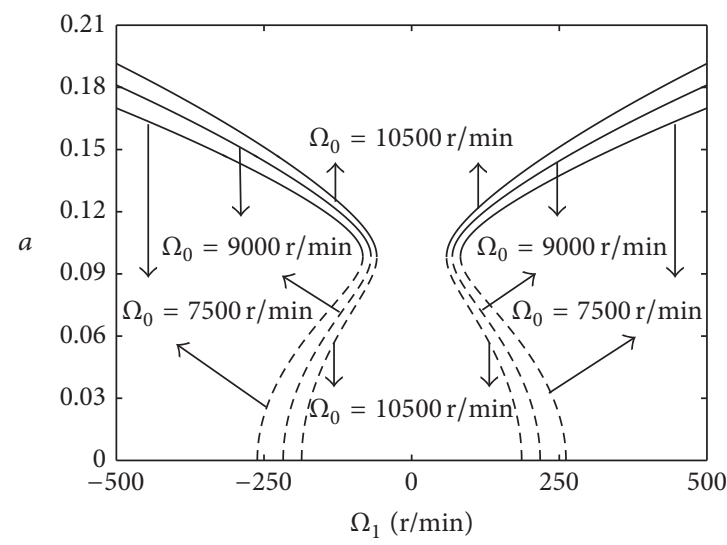

(f) $\varepsilon \sigma=-0.01, B_{0 z}=1 \mathrm{~T}$

FIGURE 6: The characteristic curves of resonance amplitude-amplitude of velocity disturbance term.

distance between different curves decreases with the increase of $\Omega_{1}$.

Figure 6 represents the characteristic curves of resonance amplitude-amplitude of velocity disturbance term with parameter $\Omega_{0}(h=8 \mathrm{~mm}, R=1 \mathrm{~m})$. It shows that unstable nontrivial solutions also existed when the detuning parameter $\varepsilon \sigma=-0.01$, while it only has stable nontrivial solutions when $\varepsilon \sigma=0$. The distance between different curves increases with the parametric $\Omega_{1}$ increasing. From Figures 4 and 5, we note that the curves show symmetrical distribution because the direction of the time-varying speed is different. Figure 6 is also shows the similar property.

Figure 7 shows the characteristic curves of resonance amplitude-amplitude of velocity disturbance term with different detuning parameter $\varepsilon \sigma\left(\Omega_{0}=7500 \mathrm{r} / \mathrm{min}, h=8 \mathrm{~mm}\right.$, $R=1 \mathrm{~m})$. From Figures $6(\mathrm{a}), 6(\mathrm{~b})$, and $6(\mathrm{c})$ we note that the unstable nontrivial solution occurs and increases when detuning parameter $\varepsilon \sigma$ increases along the negative direction. 


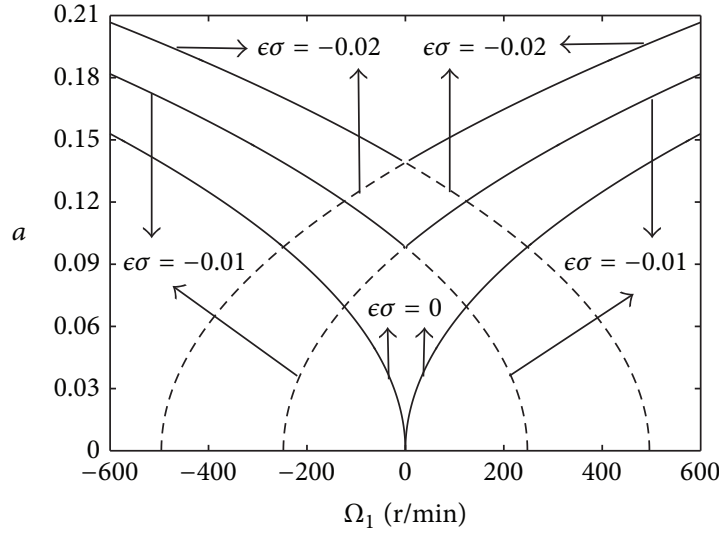

(a) $B_{0 z}=0 \mathrm{~T}$

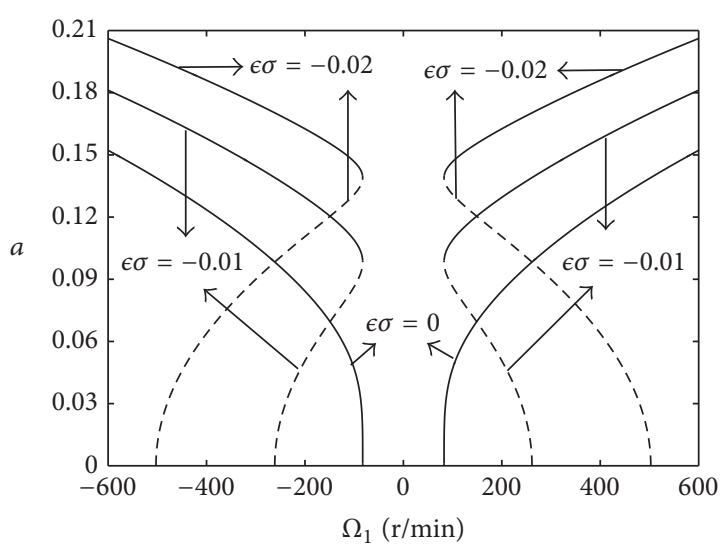

(c) $B_{0 z}=1 \mathrm{~T}$

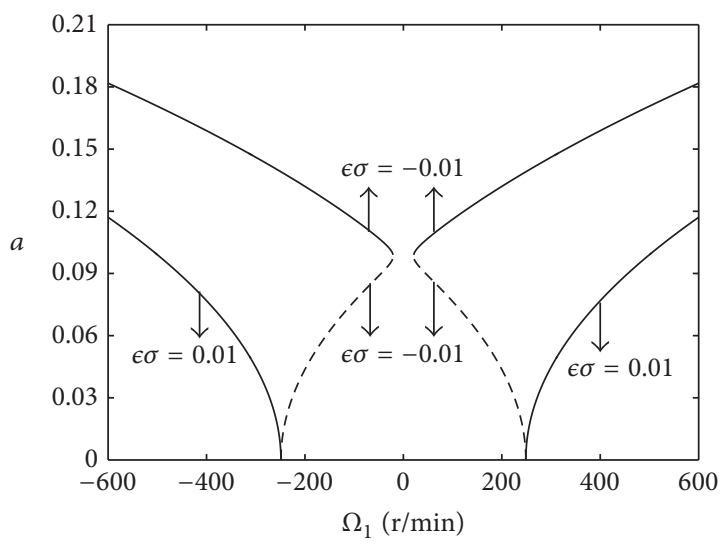

(e) $B_{0 z}=0.5 \mathrm{~T}$

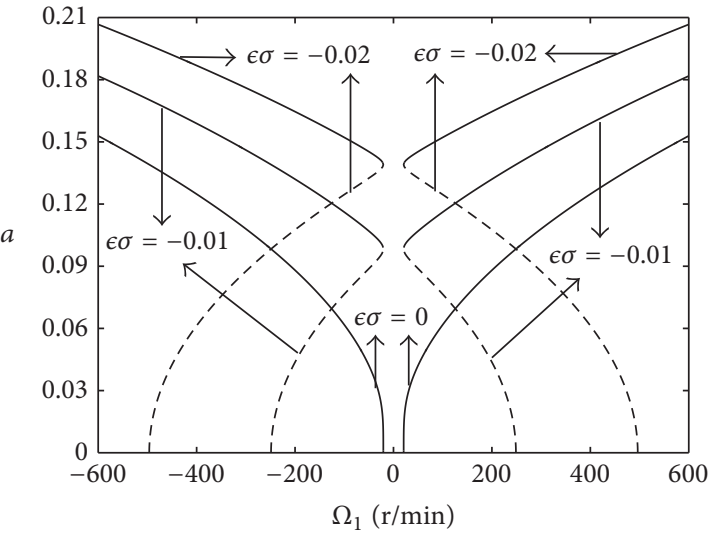

(b) $B_{0 z}=0.5 \mathrm{~T}$

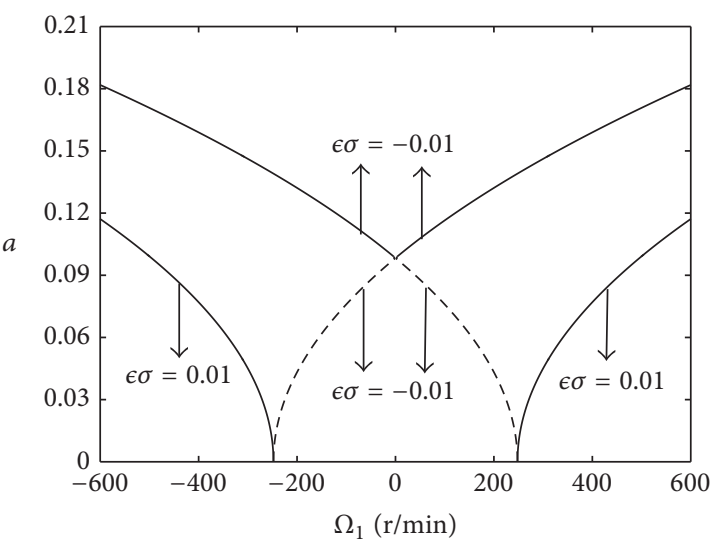

(d) $B_{0 z}=0 \mathrm{~T}$

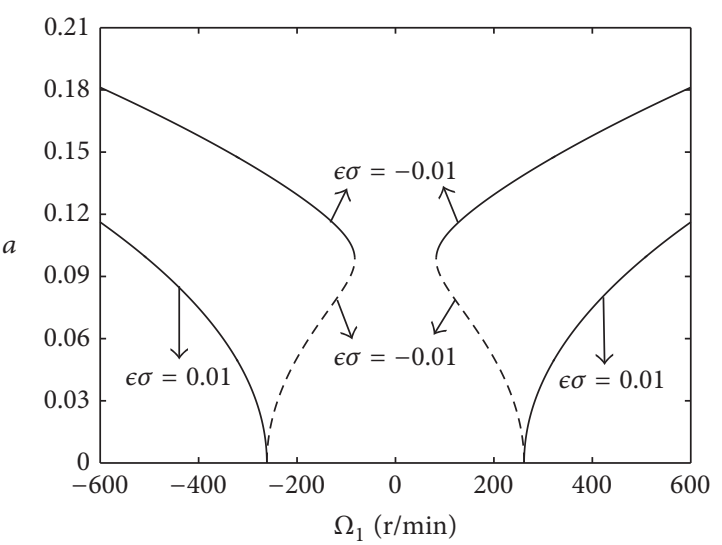

(f) $B_{0 z}=1 \mathrm{~T}$

FIGURE 7: The characteristic curves of resonance amplitude-amplitude of velocity disturbance term.

Figures 6(d), 6(e), and 6(f) represent the intersection of the stable and unstable solutions occurring on the axis of abscissa. Figures 6(a) and 6(d) show that the stable solution exists when the detuning parameter $\varepsilon \sigma$ is positive, and the zero solution region broadens with the increase of detuning parameter $\varepsilon \sigma$. These properties have no transformation with the magnetic induction intensity varying.

Figure 8 is the phase trajectory chary with different detuning parameters. The trajectory movement direction is the direction of arrow $\left(B_{0 z}=0.1 \mathrm{~T}, \Omega_{0}=7500 \mathrm{r} / \mathrm{min}, \Omega_{1}=\right.$ $400 \mathrm{r} / \mathrm{min}, R=0.8 \mathrm{~m}, h=8 \mathrm{~mm}$ ). There is only one stable solution when the detuning parameter $\varepsilon \sigma$ is close to zero, and the unstable solution occurs with the increase of $\varepsilon \sigma$ in negative direction. There only exists a stable focus $S_{1}$ in Figure 8(a) whose stable solution is $a_{S_{1}}=0.008$, which is written as $S_{1}\left(a_{S_{1}}=0.008\right)$; there is only one stable focus $S_{1}\left(a_{S_{1}}=0.018\right)$ and a saddle point $S_{2}\left(a_{S_{2}}=0.016\right)$ in Figure 8(b); there exist a stable focus $S_{1}\left(a_{S_{1}}=0.021\right)$ and 


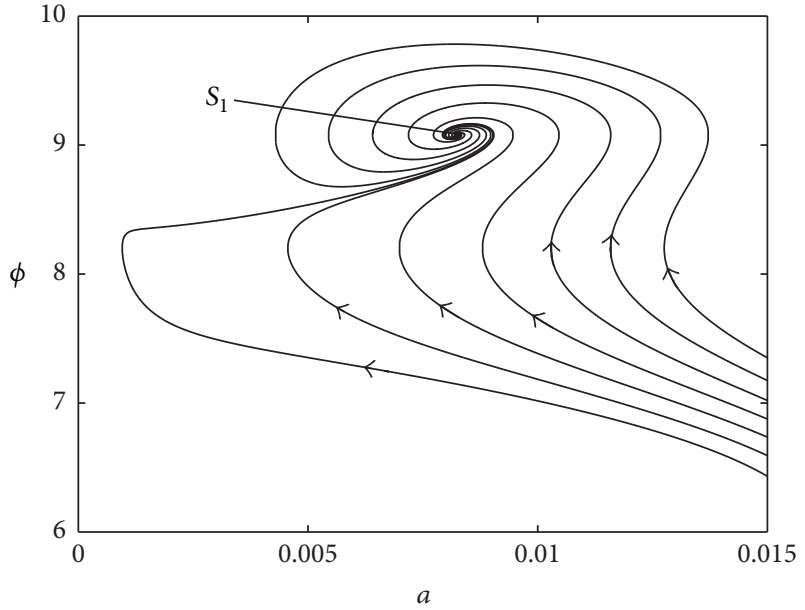

(a) $\varepsilon \sigma=0.00003$

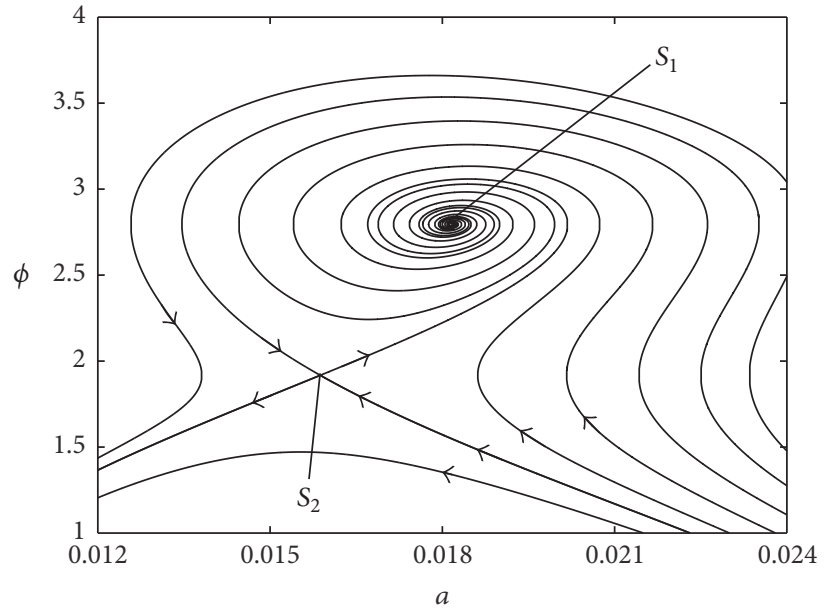

(b) $\varepsilon \sigma=-0.0003$

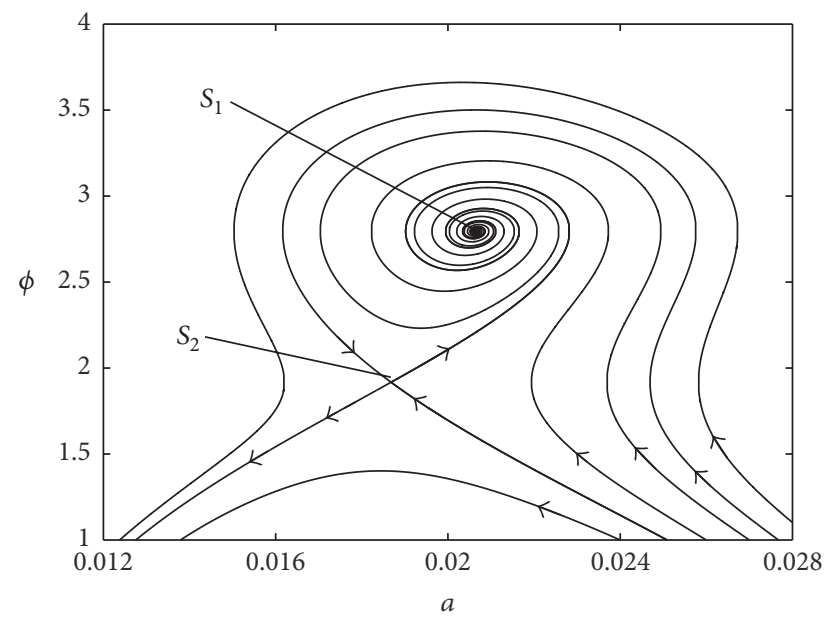

(c) $\varepsilon \sigma=-0.0004$

FIGURE 8: The phase plane curves.

a saddle point $S_{2}\left(a_{S_{2}}=0.019\right)$ in Figure 8(c). These stable solutions coincide with the amplitude characteristic curves in Figure 2.

Figure 9 is phase diagrams with different parameters. We get that the amplitude of the circular plate increases with the increase of the detuning parameter $\varepsilon \sigma$, magnetic induction intensity $B_{0 z}$, and the parametric $\Omega_{1}$ and decreases with the increase of parametric $\Omega_{0}$. The solution coincides with the amplitude characteristic curves in Figure 2. The rotating system represents single period condition. The singular point is $(0,0)$.

\section{Conclusions}

Nonlinear principal parametric resonance and stability for rotating circular plate subjected to parametric excitation resulting from the time-varying speed in the magnetic field are investigated. The influence of rotating velocity, the detuning parameter, the magnetic induction intensity, thickness, and radius on the principal parametric resonance is studied.

(1) The magnetoelastic parameter vibration equation of rotating electroconductive circular plate in the magnetic field is derived.

(2) According to the numerical examples, the following results can be concluded: (a) the resonance amplitude presents different change regularities with variation of rotating velocity, the detuning parameter, and the magnetic induction intensity; (b) there are unstable and stable solutions and they are symmetrical with $\Omega_{1}=0$; (c) the magnetic induction intensity can be chosen or adjusted so that the resonance amplitude becomes zero.

\section{Competing Interests}

The authors declare that there is no conflict of interests regarding the publication of this manuscript. 


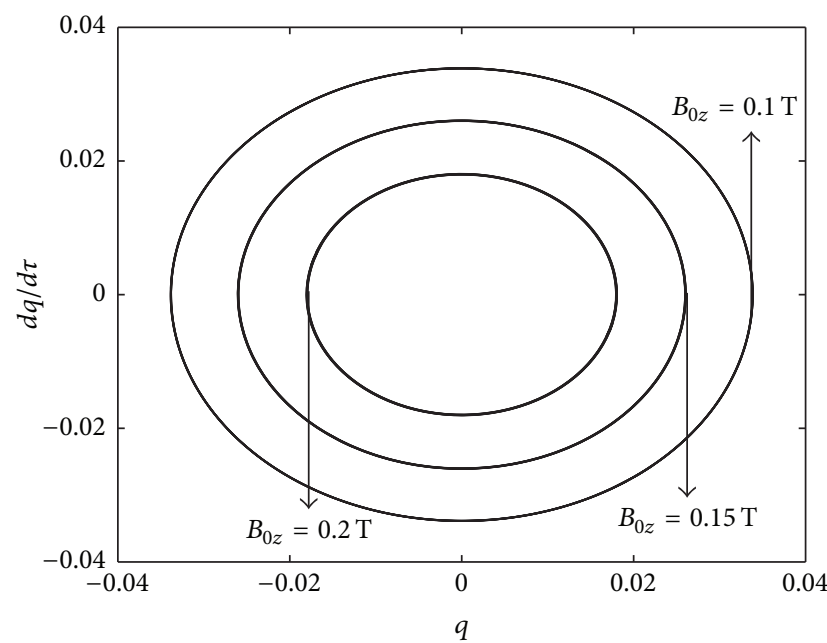

(a) $\Omega_{0}=7500 \mathrm{r} / \mathrm{min}, \Omega_{1}=400 \mathrm{r} / \mathrm{min}, \varepsilon \sigma=0.0008$

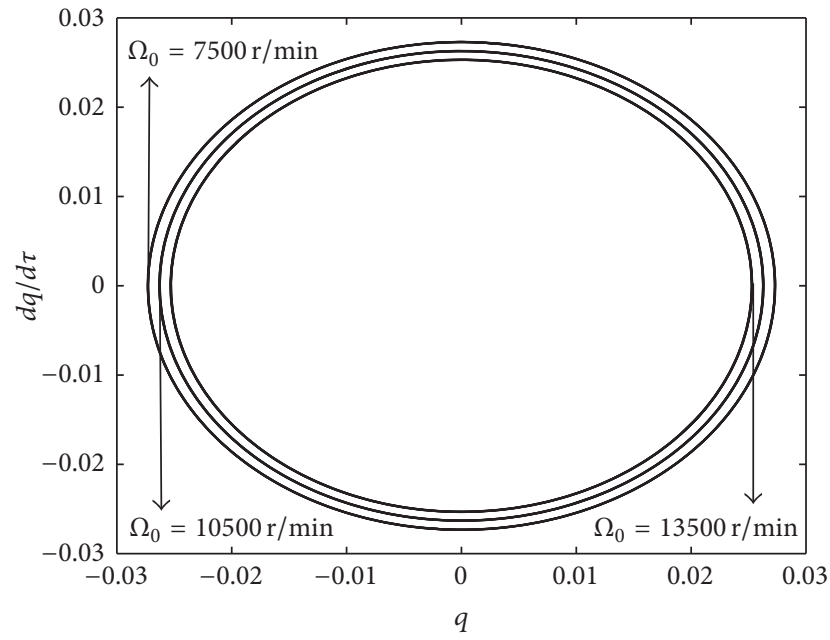

(c) $\Omega_{1}=400 \mathrm{r} / \mathrm{min}, B_{0 z}=0.1 \mathrm{~T}, \varepsilon \sigma=0.0008$

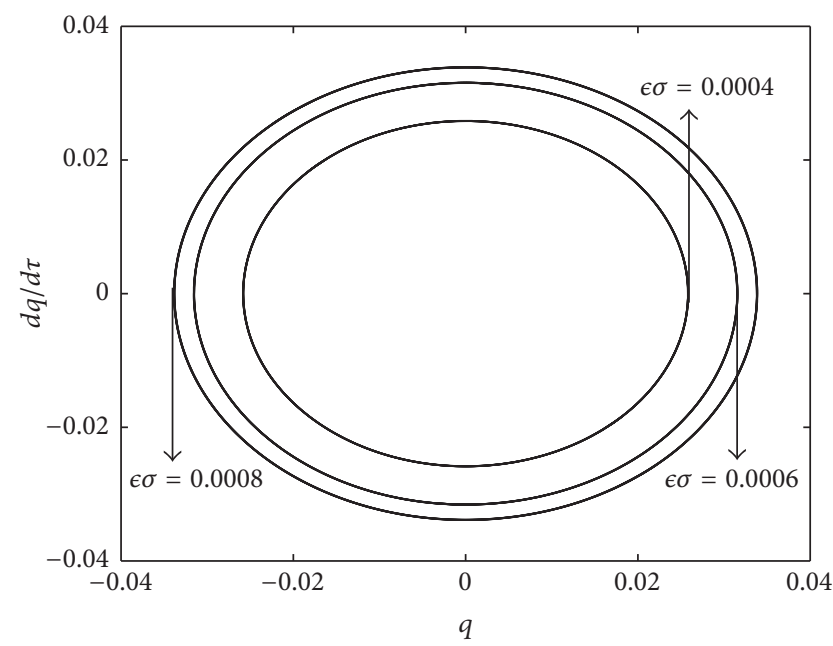

(b) $\Omega_{1}=400 \mathrm{r} / \mathrm{min}, \Omega_{0}=7500 \mathrm{r} / \mathrm{min}, B_{0 z}=0.1 \mathrm{~T}$

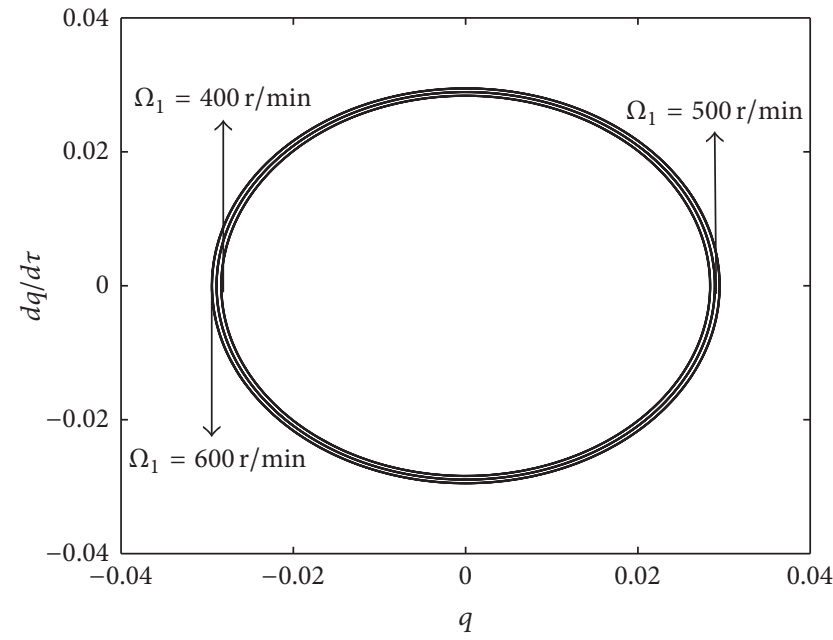

(d) $\Omega_{0}=7500 \mathrm{r} / \mathrm{min}, B_{0 z}=0.1 \mathrm{~T}, \varepsilon \sigma=0.0008$

FIgURE 9: The phase portrait.

\section{Acknowledgments}

This work was supported by the Project of National Natural Science Foundation of China (no. 11472239), Hebei Provincial Natural Science Foundation of China (no. A2015203023), The Key Project of Science and Technology Research of Higher Education of Hebei Province of China (no. ZD20131055), and 10th International Conference on Structural Integrity and Failure.

\section{References}

[1] J. Awrejcewicz, R. Starosta, and G. Sypniewska-Kamińska, "Decomposition of governing equations in the analysis of resonant response of a nonlinear and non-ideal vibrating system," Nonlinear Dynamics, vol. 82, no. 1-2, pp. 299-309, 2015.

[2] R. Starosta, G. Sypniewska-Kamińska, and J. Awrejcewicz, "Quantifying non-linear dynamics of mass-springs in series oscillators via asymptotic approach," Mechanical Systems and Signal Processing, vol. 89, pp. 149-158, 2017.
[3] S. K. Dwivedy and R. C. Kar, "Non-linear dynamics of a slender beam carrying a lumped mass under principal parametric resonance with three-mode interactions," International Journal of Non-Linear Mechanics, vol. 36, no. 6, pp. 927-945, 2001.

[4] A. F. El-Bassiouny, "Principal parametric resonances of non-linear mechanical system with two-frequency and self-excitations," Mechanics Research Communications, vol. 32, no. 3, pp. 337-350, 2005.

[5] Z. H. Feng, X. J. Lan, and X. D. Zhu, "Principal parametric resonances of a slender cantilever beam subject to axial narrowband random excitation of its base," International Journal of Non-Linear Mechanics, vol. 42, no. 10, pp. 1170-1185, 2007 (Chinese).

[6] Z. H. Feng and H. Y. Hu, "Nonlinear dynamics of flexible beams undergoing a large linear motion of basement principal parametric and internal resonances," Journal of Vibration Engineering, vol. 17, no. 2, pp. 126-131, 2004.

[7] S. H. Hashemi, S. Farhadi, and S. Carra, "Free vibration analysis of rotating thick plates," Journal of Sound and Vibration, vol. 323, no. 1-2, pp. 366-384, 2009. 
[8] Y.-D. Hu and J.-Z. Zhang, "Principal parametric resonance of axially accelerating rectangular thin plate in magnetic field," Applied Mathematics and Mechanics, vol. 34, no. 11, pp. 14051420, 2013.

[9] Y. D. Hu, L. Y. Xu, and X. Z. Bai, "The axial symmetric vibration of circular plate in transverse magnetic field," Journal of Vibration and Shock, vol. 17, no. 4, pp. 71-74, 1998 (Chinese).

[10] Y. Hu and Y. Zhang, "Analysis of magneto-elastic principal resonance of axially moving current-conducting plate in magnetic field," Journal of Mechanical Engineering, vol. 49, no. 23, pp. 123128, 2013.

[11] Y.-D. Hu and G.-J. Du, "Forced vibration of a thin round conductive plate in magnetic field," Engineering Mechanics, vol. 24, no. 7, pp. 184-188, 2007 (Chinese).

[12] B. Özhan and M. Pakdemirli, "Principal parametric resonances of a general continuous system with cubic nonlinearities," Applied Mathematics and Computation, vol. 219, no. 5, pp. 24122423, 2012.

[13] M. Shahgholi and S. E. Khadem, "Primary and parametric resonances of asymmetrical rotating shafts with stretching nonlinearity," Mechanism and Machine Theory, vol. 51, pp. 131-144, 2012.

[14] J. Awrejcewicz, L. Kurpa, and O. Mazur, "On the parametric vibrations and meshless discretization of orthotropic plates with complex shape," International Journal of Nonlinear Sciences and Numerical Simulation, vol. 11, no. 5, pp. 371-386, 2010.

[15] J. Awrejcewicz, A. V. Krysko, I. E. Kutepov, N. A. Zagniboroda, M. V. Zhigalov, and V. A. Krysko, "Analysis of chaotic vibrations of flexible plates using fast Fourier transforms and wavelets," International Journal of Structural Stability and Dynamics, vol. 13, no. 7, Article ID 1340005, 2013.

[16] J. Awrejcewicz, I. V. Papkova, E. U. Krylova, and V. A. Krysko, "Wavelet-based analysis of the regular and chaotic dynamics of rectangular flexible plates subjected to shear-harmonic loading," Shock and Vibration, vol. 19, no. 5, pp. 979-994, 2012.

[17] Y.-Q. Tang and L.-Q. Chen, "Stability analysis and numerical confirmation in parametric resonance of axially moving viscoelastic plates with time-dependent speed," European Journal of Mechanics-A/Solids, vol. 37, pp. 106-121, 2013.

[18] Y. Zhao, T. Wang, and H. J. Kang, "Analysis of the stability of principal parametric resonance of stayed-cable," Journal of Dynamics and Control, vol. 6, no. 2, pp. 112-116, 2008 (Chinese).

[19] Y.-H. Zhou, Y. Gao, and X. J. Zheng, "Buckling and postbuckling analysis for magneto-elastic-plastic ferromagnetic beam-plates with unmovable simple supports," International Journal of Solids and Structures, vol. 40, no. 11, pp. 2875-2887, 2003.

[20] A. H. Nayfeh and D. T. Mook, Nonlinear Oscillations, John Wiley \& Sons, New York, NY, USA, 1979. 


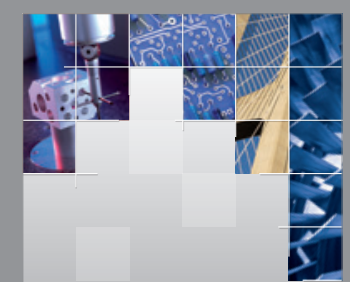

\section{Enfincering}
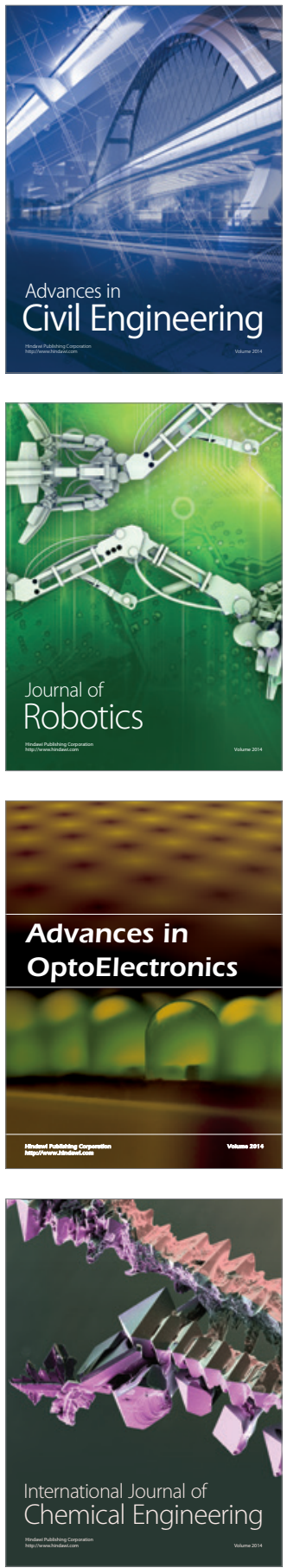

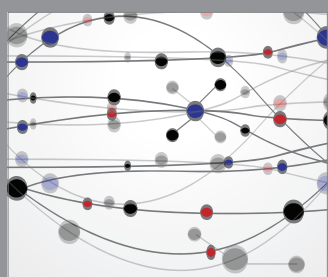

The Scientific World Journal

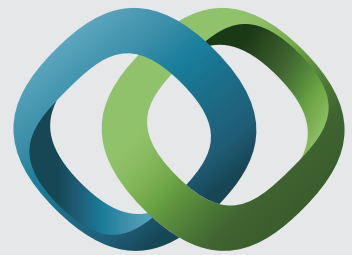

\section{Hindawi}

Submit your manuscripts at

https://www.hindawi.com
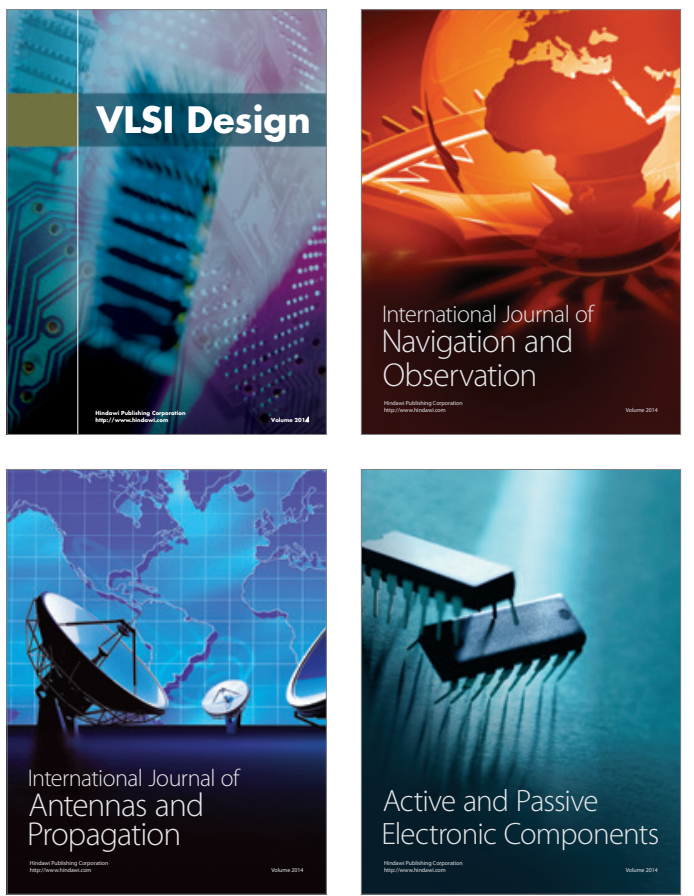
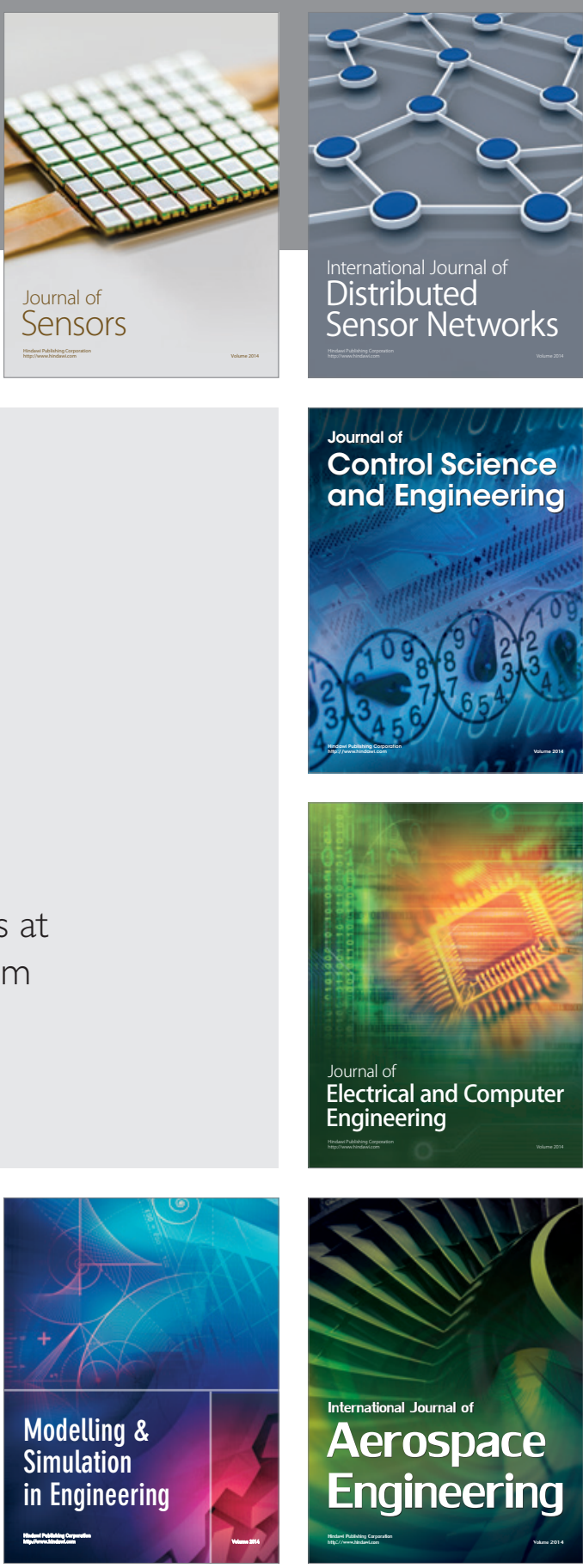

International Journal of

Distributed

Sensor Networks

$-$

Joumal of

Control Science

and Engineering
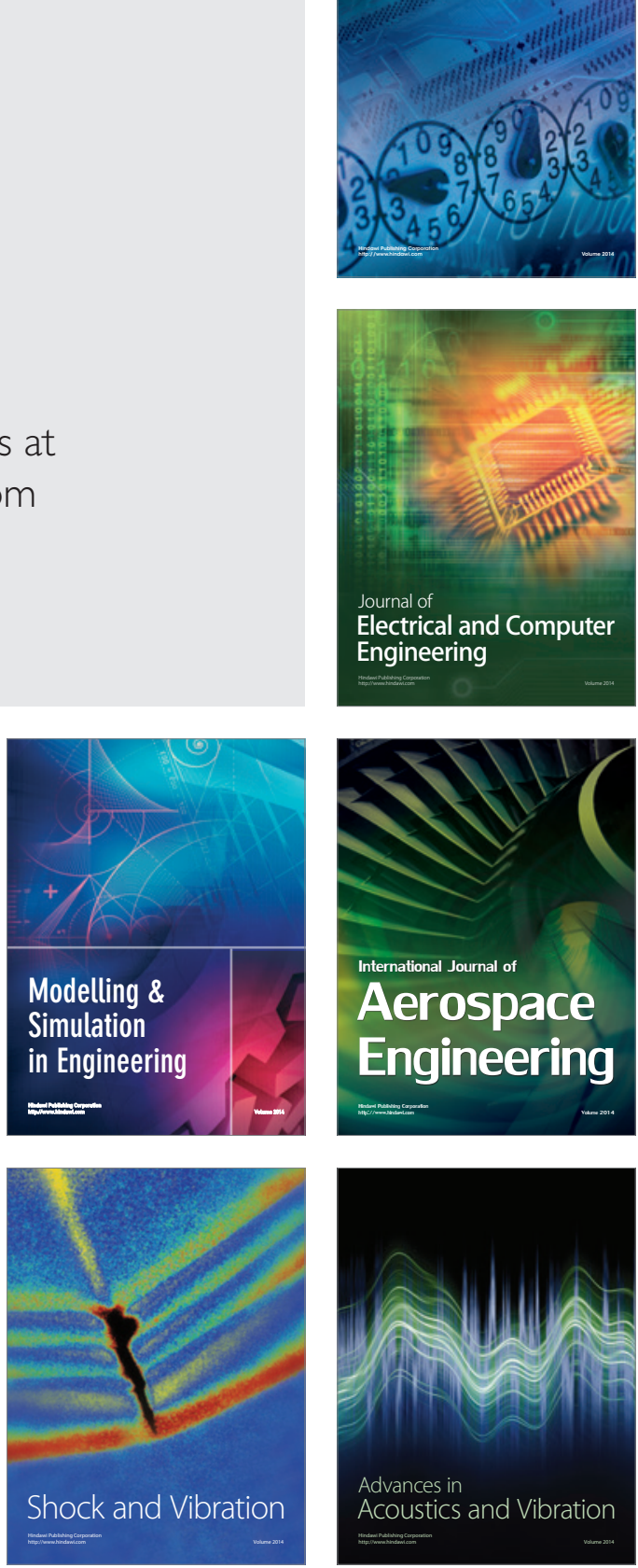